DIVISION OF THE HUMANITIES AND SOCIAL SCIENCES

CALIFORNIA INSTITUTE OF TECHNOLOGY

PASADENA, CALIFORNIA 91125

INDECISION THEORY: QUALITY OF INFORMATION AND VOTING BEHAVIOR

Paolo Ghirardato

Jonathan N. Katz

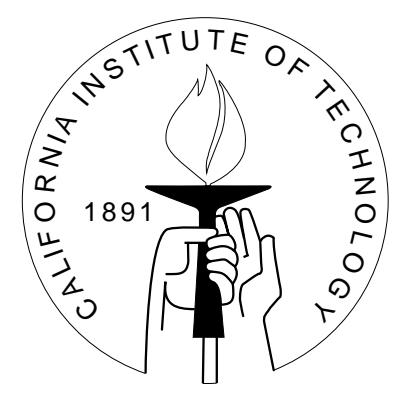

SOCIAL SCIENCE WORKING PAPER 1106R 


\title{
Indecision Theory: Quality of Information and Voting Behavior*
}

\author{
Paolo Ghirardato $\quad$ Jonathan N. Katz ${ }^{\S}$
}

\begin{abstract}
In this paper we show how to incorporate quality of information into a model of voting behavior. We do so in the context of the turnout decision of instrumentally rational voters who differ in their quality of information, which we refer to as ambiguity. Ambiguity is reflected by the fact that the voter's beliefs are given by a set of probabilities, each of which represents in the voter's mind a different possible scenario.

We show that in most elections voters who satisfy the Bayesian model do not strictly prefer abstaining over voting for one of the candidates. In contrast, a voter who is averse to ambiguity considers abstention strictly optimal when the candidates' policy positions are both ambiguous and they are "ambiguity complements". Abstaining is preferred since it is tantamount to mixing the prospects embodied by the two candidates, thus enabling the voter to "hedge" the candidates' ambiguity.
\end{abstract}

\section{Introduction}

Of all the Californians who turned out at the polls on Election Day 1998, only $2.74 \%$ did not express an opinion on who should be the next Governor. In contrast, $24.65 \%$ of them abstained on the election of the Superintendent of Public Instruction, and 31.56\% abstained on the approval vote of a California Supreme Court Judge. As to propositions

*This paper replaces an earlier paper that was circulated in August 1997 with the title "Indecision Theory: An Informational Model of Roll-Off". We are very grateful to Alessandro Lizzeri for many detailed comments, and to Doug Bernheim, Peter Bossaerts, Colin Camerer, Tim Feddersen, Michel Le Breton, Tom Palfrey, Ken Shotts and audiences at Caltech, UCLA, the Department of Political Science at UC-San Diego, the 1999 Wallis Conference on Political Economy and the 2001 SITE Conference for helpful comments and discussion. Jonathan Katz thanks the John M. Olin Foundation for a Faculty Fellowship supporting his research. URLs: http://www.hss.caltech.edu/ paolo/ghiro.html and http://jkatz.caltech.edu.

§D.H.S.S. (228-77), California Institute of Technology, Pasadena, CA 91125. paolo@hss.caltech.edu

`D.H.S.S. (228-77), California Institute of Technology, Pasadena, CA 91125. jkatz@caltech.edu 
on the ballot, abstention was a low $5.3 \%$ of the actual voters on the much publicized and discussed Proposition 5 - which permitted gambling at tribal casinos. In contrast, $15.23 \%$ of the actual voters abstained on Proposition 11 - a relatively obscure legislative constitutional amendment to allow cities and counties to enter into sales tax revenue sharing agreements by a two-thirds vote of each jurisdiction's governing body. In the same election, only $1.24 \%$ of the voters of the Fourth Congressional district abstained on the Gubernatorial election, but 5.95\% abstained from voting on their district's House Representative. A similar pattern of relative abstention can be seen in the other Congressional Districts. $^{1}$

The message we draw from these data is twofold: i) voters abstain even when voting is costless (since they are already in the ballot booth); ii) abstention follows a systematic pattern, as voters are more likely to abstain in smaller races, such as local judge or school board, or less publicized initiatives. This phenomenon of "selective abstention" is not peculiar to California, it is pervasive. It is a puzzle given the "rational choice" tenets that voters are more likely to vote the lower their costs of voting and the higher their probability of casting a pivotal vote. Overwhelmingly, the voters discussed in the foregoing paragraph vote on those elections on which more information is publicly available (say, those with larger media coverage), which typically have a larger electorate and therefore a lower probability of casting a pivotal vote. They do not vote in smaller elections, which they can affect with much higher probability. That is, voters abstain in elections that they feel they have poorer information about.

The question we address in this paper is how to incorporate quality of information into a model of voting behavior. This places the paper in the larger research agenda of understanding how voters' information affects voting behavior and ultimately the outcome of political action. The most direct precursors of this paper are those of Feddersen and Pesendorfer $(1996,1999)$, which investigate how asymmetries in information across voters affect voting behavior and election results. In contrast, we focus our attention on how the quality of the information of an individual voter affects his voting choices. ${ }^{2}$

Our point of departure is the observation that the standard Bayesian model is not well suited to modelling the differences in the quality of information that the voter has about different elections. This has been known at least ever since the seminal paper of Ellsberg (1961), which made clear that quality of information — or, in Ellsberg's term, "ambiguity" - affects the choices of most agents. Typically, agents tend to undervalue the more "ambiguous" options, i.e., the options whose stochastic structure the agent knows less about. This pattern of behavior has been dubbed "ambiguity aversion", and its empirical relevance has been further demonstrated by a large number of experiments (see Luce (2000) for a survey). ${ }^{3}$ However, it conflicts with the standard Bayesian model,

\footnotetext{
${ }^{1}$ All the data come from the Statement of Vote: General Election, November 3, 1998 by California Secretary of State Bill Jones (which can be found at http://vote98.ss.ca.gov/).

${ }^{2}$ Throughout, we use masculine pronouns for voters and feminine pronouns for candidates.

${ }^{3}$ As to its normative relevance, we remark that many agents who make ambiguity averse choices do so without repentance. Including, as Ellsberg (1961) reports, L.J. Savage, the father of the Bayesian
} 
Savage (1954)'s "subjective expected utility" (SEU) model. An agent that satisfies SEU cannot care about ambiguity.

We consider voters who are "instrumentally rational" — i.e., they only care about the policy that is eventually implemented. We show that in most political elections voters who satisfy the Bayesian model do not strictly prefer abstaining over voting for one of the candidates. ${ }^{4}$ In contrast, an ambiguity averse voter does strictly prefer to abstain on those elections whose policy results he considers more ambiguous. Roughly, the reason for this preference is the following. Ambiguity is reflected by the fact that the voter's beliefs are given by a set of probabilities, each of which represents in the voter's mind a different possible scenario. The voter's aversion to such ambiguity is reflected by the fact that he evaluates each available option by using the worst possible scenario for that option - that is, he satisfies the "maxmin expected utility with multiple priors" (MEU) model of Gilboa \& Schmeidler (1989). We find that the voter considers abstention strictly optimal when the candidates' policy positions are both ambiguous, and they are "ambiguity complements": One candidate looks better than the other in some scenario he envisions, while the opposite happens in another scenario. That is, the voter is afraid that voting for each candidate will turn out to be a "mistake" and voting for the other one would have been the right thing to do. Abstaining is preferred as it is tantamount to mixing the prospects embodied by the two candidates, thus enabling the voter to "hedge" the candidates' ambiguity. This rationalization of abstention is not possible if the voter satisfies SEU. An SEU voter sees no need to hedge since he does not mind ambiguity. ${ }^{5}$

Ambiguity is a common aspect of any decision problem, and in most situations it is reasonable to believe that agents are averse to such ambiguity. However, there is an additional reason to believe that ambiguity aversion plays an important role in voting decisions. In an enlightening paper on the psychology of people's reaction to ambiguity, Fox \& Tversky (1995) find ample evidence to support what they call the "comparative ignorance" hypothesis: Agents are more ambiguity averse when asked to compare ambiguous and unambiguous actions together than when asked to evaluate ambiguous actions in isolation. Further, agents are more ambiguity averse when asked to evaluate ambiguous actions knowing that more informed agents are making a similar evaluation. Their findings suggest that an agent who faces multiple decision problems - and has plenty of reliable information on some problems and little reliable information on some others - displays stronger ambiguity aversion in those problems about which he is more poorly informed. In our case this implies that because a voter is faced with multiple elections on the ballot, he is ambiguity averse on those elections for which his information is poor and he thus is more likely to strictly prefer abstention on those elections. The presence of multiple items makes it more likely for ambiguity aversion to appear, as it

model.

${ }^{4}$ The exception include those elections in which the assumptions of Feddersen \& Pesendorfer (1999) are satisfied.

${ }^{5}$ One of the assumptions we make in our main model implies that no SEU voter strictly prefers to abstain, thus showing that the explanation we offer is independent of any explanation based on SEU voters. 
makes the agent aware of his relative ignorance.

In evaluating our contribution to the theory of voting behavior, it is important to understand its relation to the well-known "voting paradox". If going to the polls entails some small but positive fixed cost, why would an instrumentally rational voter decide to show up for elections with a large number of voters? The voter's benefit from voting depends on the probability that his vote is pivotal, which in those elections is ridiculously small. In this paper, we do not address this voting paradox. We assume that voting carries zero fixed costs, so that the voter goes to the polls if he wants to vote on some election regardless of his probability of affecting that election. However, it would be straightforward to extend our model to include a fixed cost to voting by following the traditional explanation of turnout of Riker \& Ordeshook (1968). ${ }^{6}$ We could assume that the voters who go to the polls are those whose fixed cost is offset by a benefit due to the fact that they think it is their civic duty to do so. Once there, they satisfy the conditions of our model and they only vote on those elections in which they feel sufficiently confident of their choice. ${ }^{7}$ Of course, a more general model of voting behavior might be developed which allows ambiguity aversion to explain turnout and selective abstention without the ad hoc civic duty assumption, but the development of such model goes well beyond the first step that we want to take here.

To the best of our knowledge, there are not many papers that discuss formally how information affects the decision to vote in an election. All assume that voters are Bayesian, so that as argued above their explanations of a voter's abstention are complementary to the one developed here. In Section 7 we present a more detailed comparison with these alternative explanations.

There exists a flourishing literature on the consequences of ambiguity aversion for other economic behavior, using either the MEU model or a closely related model - the "Choquet expected utility" model of Schmeidler (1989). For instance, ambiguity aversion provides useful insights in the study of diverse phenomena, such as the equity premium and home bias puzzles in asset pricing (e.g.: Epstein \& Wang (1994), Epstein \& Miao (2000), Hansen, Sargent \& Tallarini (1999)) or the existence of incomplete contracts (Mukerji (1998)). This paper is, again to the best of our knowledge, the first attempt to use ambiguity aversion to study a political economy issue. The choice of voting is a natural first step, for it clearly seems to be a decision problem in which low quality of information is the norm rather than the exception.

The paper proceeds as follow. We set the stage by introducing basic notation and the set-up in Section 1. In Section 2, we assume that the voter satisfies the SEU model and show the conditions that his beliefs must satisfy in order for abstention to be strictly optimal. We then generalize to the MEU model. In Section 3 we present an example of a MEU voter who strictly prefers to abstain. Section 4 analyzes in depth why such

\footnotetext{
${ }^{6}$ See Aldrich (1993) for a review of the literature on the voting paradox.

${ }^{7}$ Indeed, civic duty might be an additional factor contributing to a voter's ambiguity aversion, by making him more aware of the societal costs of a wrong vote.
} 
a voter abstains, thus shedding some light on the relation between ambiguity aversion and abstention. Section 5 briefly discusses the consequences of our model for voting in multiple elections. Section 6 provides some extensions of the model used in the previous two sections, showing that our results do not depend on the assumptions we make there (with the exception of instrumental rationality and the MEU decision rule). Section 7 closes with a brief discussion of some alternative explanations of selective abstention.

\section{The Voting Problem}

We imagine a voter in the polling booth holding a blank ballot in his hands. All "fixed costs" of voting are already sunk, and the only cost that the voter is facing is that of making up his mind on each item on the ballot. In general, we are interested in describing his behavior if the ballot requires him vote on, say, $M$ different elections. Which elections is he going to vote on, and which ones is he going to abstain on? Which candidate is he going to vote for if he does not abstain? To do so, we consider the voter's behavior in each single election, given his knowledge on the issues and candidates at the moment in which he is looking at the blank ballot. While strictly speaking our analysis is independent of the existence of multiple issues on the ballot, we have suggested earlier the possible role that such multiplicity plays, via the comparative ignorance hypothesis, in motivating the conditions that deliver the optimality of abstention in some elections.

Fix one election with two candidates $A$ and $B .{ }^{8}$ The voter's decision problem is described by the following: His possible choices, the state space describing the relevant uncertainty, and the possible outcomes of his choice.

As to the outcomes, following the tradition in the literature on voting we assume that the voter is instrumentally rational; that is, he only cares about the policy which is eventually implemented: If we let $X$ denote the set of the possible policy positions that a candidate can take, the voter is only interested in what policy $x \in X$ is eventually implemented:

Assumption 1 The final utility to the voter of the elected candidate implementing policy $x \in X$ is given by $u(x)$, where $u: X \rightarrow[0,1]$.

This assumption implies that the voter's final utility of seeing any policy $x$ is independent of the election results and (the voter's conjectures about) the loser's policy position. Such independence rules out, for instance, the possibility that each of the choices in a election might carry a "signalling" value for a voter; that is, his utility might be affected by his action as well as the eventual policy outcome. Since the voter's utility function is later assumed to be cardinal, the normalization within $[0,1]$ is tantamount to assuming that

\footnotetext{
${ }^{8}$ While we stick to the standard case of two candidates in order to keep notation to a minimum (and to draw pictures on a two-dimensional page), nothing in the analysis to be presented depends on having two candidates. All results immediately generalize to the case of more than two candidates.
} 


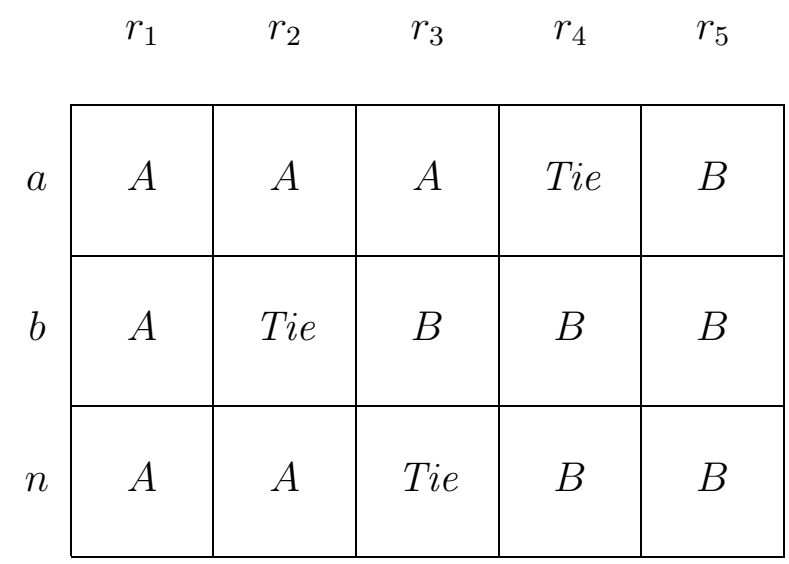

Table 1: Election Outcomes

$u$ is bounded. It implies that the unit square $[0,1]^{2}$ contains all the conceivable pairs of utility levels for the voter, as it corresponds to the set of all the pairs $(u(x), u(y))$, where $x \in X$ (resp. $y \in X$ ) is candidate $A$ 's (resp. B's) real policy position.

When in the booth, the voter is uncertain about the outcome of the election in the absence of his vote. Let $N_{j}$ denote the number of votes cast by all other voters in favor of candidate $j \in\{A, B\}$ (abstentions are of course allowed; i.e., we do not require that $N_{A}+N_{B}$ be equal to the number of eligible voters minus 1 ). Then the set of possible election results is given by $R=\left\{r_{1}, r_{2}, r_{3}, r_{4}, r_{5}\right\}$, where $r_{1}$ is the event that $N_{A}>N_{B}+1$, $r_{2}$ is $N_{A}=N_{B}+1, r_{3}$ is $N_{A}=N_{B}, r_{4}$ is $N_{A}=N_{B}-1$, and $r_{5}$ is $N_{A}<N_{B}-1$. Thus the event that this particular voter is pivotal in the election is given by a subset of $\left(r_{2} \cup r_{3} \cup r_{4}\right)$, depending on which tie-breaking rule is adopted. ${ }^{9}$ Table 1 summarizes these considerations by plotting election outcomes as a result of $r$ and the of the voter's choice of voting for $A$ (denoted by $a$ ), voting for $B$ (denoted by $b$ ), or abstaining (denoted by $n$ ). The voter's choice set is thus $\{a, b, n\}$.

The entries of the matrix do not complete resolve the voter's uncertainty, since the voter cares about the policy that the elected candidate implements. Thus, his uncertainty would be fully resolved only if he knew the real policy positions of both candidates, a pair belonging to the set $S \equiv X^{2}$. Adding this second dimension to the voter's uncertainty, we see that the voter's state space is given by $T \equiv R \times S$, with typical element $t=(r, s)=$ $\left(r, s_{1}, s_{2}\right)$. Coupled with the voter's choice, a state $t$ determines a policy outcome $x \in X$ (which by assumption 1 is what determines the voter's final utility) once a tie-breaking rule for the election is specified. We follow Feddersen \& Pesendorfer $(1996,1999)$ in making the following:

Assumption 2 In case of tie, candidate A wins.

As we argue in Section 6, the nature of our results does not change (though some condi-

\footnotetext{
${ }^{9}$ For instance, if the tie-breaking rule is that candidate $A$ wins in case of a tie, then the set of election results in which the voter is pivotal is $\left(r_{3} \cup r_{4}\right)$.
} 


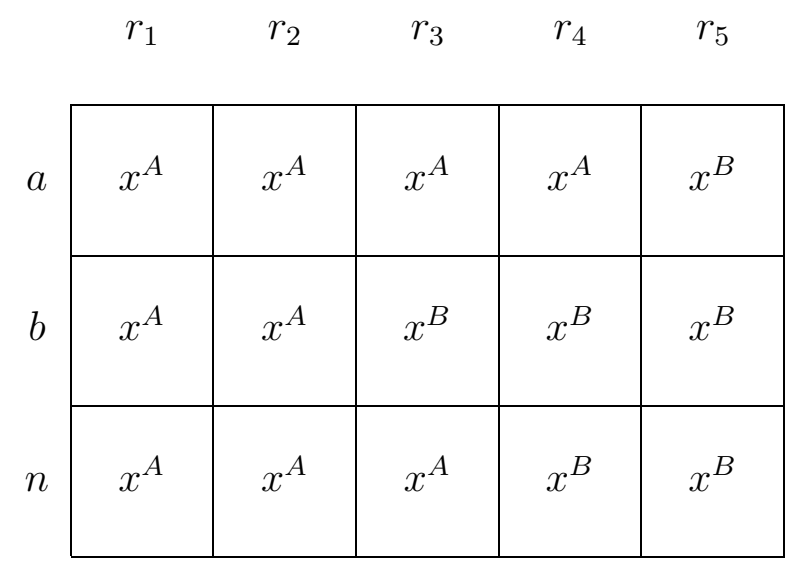

Table 2: Election "Payoffs"

tions differ) if we break ties by a different rule, say the toss of a coin. In Table 2 we use this tie-breaking rule to rewrite the profiles of Table 1 in terms of the voter's "payoffs", rather than of the election outcomes. The payoffs are given by the candidates' policy positions, which from the perspective of the voter are random variables on $S$ (hence the quotes). Specifically, they are $x^{A}: S \rightarrow X$ and $x^{B}: S \rightarrow X$ defined as follows: For each policy pair $s=\left(s_{1}, s_{2}\right) \in S, x^{A}(s)=s_{1}$ and $x^{B}(s)=s_{2}$.

\section{Do SEU Maximizers Strictly Prefer to Abstain?}

We now address the question of whether SEU maximizers may strictly prefer abstaining over voting for one of the candidates. This is a rhetorical question, because we already know from Feddersen \& Pesendorfer (1996) that the answer is yes. Here we take a more decision-theoretic perspective and look at the type of beliefs that a SEU maximizer must hold (say, in a given equilibrium) for abstention to be his strictly optimal choice.

A voter who satisfies assumption 1 chooses according to the SEU model if there is a cardinal utility $u$ on $X$ and a unique probability measure $\pi$ representing the voter's beliefs on the state space $T,{ }^{10}$ such that the voter chooses the act $f \in\{a, b, n\}$ which maximizes

$$
U(f)=\int_{T} u(f(t)) d \pi(t) .
$$

\footnotetext{
${ }^{10}$ To be more precise, assume that the policy events in $X$ are elements of a field $\mathcal{F}_{X}$, and that the election events are all the subsets of $R$ (i.e., any element of the power set $2^{R}$ ). The voter's beliefs are given by a finitely additive probability measure $\pi: \mathcal{F} \rightarrow[0,1]$, where $\mathcal{F}=2^{R} \otimes\left(\mathcal{F}_{X} \otimes \mathcal{F}_{X}\right)$, the product field of $2^{R}$ and $\mathcal{F}_{X} \otimes \mathcal{F}_{X}$, in turn the product field of $\mathcal{F}_{X}$ with itself. We henceforth denote by $\Delta(T)$ the set of all the probability measures with these properties. Similarly, we denote by $\Delta(S)$ (resp. $\Delta(R)$, resp. $\Delta(X)$ ) the set of the finitely additive probability measures on the product field $\mathcal{F}_{X} \otimes \mathcal{F}_{X}$ (resp. the field $2^{R}$, resp. the field $\mathcal{F}_{X}$ ).
} 


\subsection{Conditions for Abstention under SEU}

We now show that a SEU maximizing voter will abstain if and only if he thinks that the pivotal states $r_{3}$ and $r_{4}$ are both possible, and his beliefs conditional on these election results reflect a "positive" correlation between election results and desirability of the policy position of the elected candidate.

To start, it is helpful to reformulate the expected utility of each choice $U(f)$ with the help of some additional notation. Given a voter's belief $\pi$, let $\lambda$ denote the marginal of $\pi$ over the first coordinate of the state space, ${ }^{11}$ and for each $i=1, \ldots, 5$, let $\lambda_{i} \equiv \lambda\left(\left\{r_{i}\right\}\right)$. That is, $\lambda_{i}$ is the voter's subjective probability that election result $r_{i}$ will obtain. Denote by $\mu_{i}$ the conditional probability on $S$ induced by $\pi$ in election result $r_{i}{ }^{12}$ and let $\mu_{i}^{A}$ (resp. $\mu_{i}^{B}$ ) denote the marginal of $\mu_{i}$ on its first (resp. second) coordinate. ${ }^{13}$ That is, $\mu_{i}^{A}$, say, is the voter's subjective probability distribution over the possible policies of candidate $A$ conditional on election result $r_{i}$. Finally, given a belief $\sigma \in \Delta(X)$ on the possible policy positions of a single candidate and a function $u$, let $v(\sigma)$ denote the voter's expected utility of having that candidate in office. That is,

$$
v(\sigma) \equiv \int_{X} u(x) d \sigma(x)
$$

Clearly, $v(\sigma) \in[0,1]$. Looking at Table 2 and using this notation with the measures $\mu_{i}^{A}$ and $\mu_{i}^{B}$, we can rewrite $U(a)$ as follows:

$$
U(a)=\sum_{i=1}^{4} \lambda_{i} v\left(\mu_{i}^{A}\right)+\lambda_{5} v\left(\mu_{5}^{B}\right) .
$$

$U(b)$ and $U(n)$ are similarly rewritten, mutatis mutandis. Now, $U(n)>U(a)$ if and only if

$$
\lambda_{4} v\left(\mu_{4}^{A}\right)<\lambda_{4} v\left(\mu_{4}^{B}\right) .
$$

On the other hand, $U(n)>U(b)$ if and only if

$$
\lambda_{3} v\left(\mu_{3}^{A}\right)>\lambda_{3} v\left(\mu_{3}^{B}\right) .
$$

We are thus able to draw the following conclusion:

Proposition 1 Suppose that assumptions 1 and 2 hold, and that the voter has SEU preferences with utility $u$ and beliefs $\pi$. Then he strictly prefers abstaining to voting for either candidate if and only if he deems each of the "pivotal states" $r_{3}$ and $r_{4}$ possible and his beliefs conditional on state $r_{3}$ favor candidate $A$, while his beliefs conditional on state $r_{4}$ favor candidate $B$. (Formally, he strictly prefers to abstain if and only if (i) $\lambda_{i}>0$ for $i=3,4$, (ii) $v\left(\mu_{3}^{A}\right)>v\left(\mu_{3}^{B}\right)$, and (iii) $v\left(\mu_{4}^{A}\right)<v\left(\mu_{4}^{B}\right)$.)

\footnotetext{
${ }^{11}$ That is, for every event $C \in 2^{R}$, let $\lambda(C)=\pi(C \times X \times X)$.

${ }^{12}$ That is, for every event $D \in\left(\mathcal{F}_{X} \otimes \mathcal{F}_{X}\right)$ let $\mu_{i}(D)=\pi\left(D \mid r=r_{i}\right)$.

${ }^{13}$ That is, for every event $E \in \mathcal{F}_{X}$, let $\mu_{i}^{A}(E)=\mu_{i}(E \times X)\left(\right.$ resp. $\left.\mu_{i}^{B}(E)=\mu_{i}(X \times E)\right)$.
} 
It can be easily seen from the inequalities above that if the voter assigns positive probability to at most one of the two pivotal states, he is indifferent between abstaining and voting for one of the candidates. This explains condition $(i)$.

When each pivotal state is possible, the two inequalities above are equivalent to conditions $(\mathrm{ii})$ and $(\mathrm{iii})$. That is, the voter strictly prefers to abstain if he voter interprets the fact that one more of the other voters is casting his vote for candidate $B$ as a signal that candidate $B$ is more likely to adopt a more desirable policy position for the voter, and he interprets a tie as a signal that $A$ is better (this is because of the tie-breaking rule, of course). As anticipated above, the voter's beliefs conditional on $r_{3}$ and $r_{4}$ reflect a (strictly) "positive" correlation between election results and policy positions.

These conditions are satisfied by a less informed voter in any symmetric equilibrium of the voting game of Feddersen \& Pesendorfer (1999). In particular, the fact that such voter assigns positive probability to each of the pivotal states follows from their assumption that the population of voters follows a Poisson distribution (so that every voter has a positive chance of being the only one at the polls). The fact that he has such beliefs in equilibrium follows from the assumptions that all voters' preferences are aligned in a certain fashion, and that there is a continuum of voter types and a discrete set of states.

Abstention cannot be strictly optimal if the voter believes the expected utility of a candidate to be "negatively correlated" to her election performance, or simply if he considers the election results in $R$ and the policy positions in $S$ stochastically independent. In fact, in the latter case $\mu_{3}=\mu_{4}$ so that conditions ( $\left.i i\right)$ and (iii) cannot both hold. ${ }^{14}$ Thus, if a SEU voter's beliefs satisfy stochastic independence of $R$ and $S$, he cannot strictly prefer to abstain. We henceforth assume stochastic independence, so as to make clear that the explanation of selective abstention suggested below is logically independent of any explanation tailored for SEU voters, as that of Feddersen \& Pesendorfer $(1996,1999)$. Of course, stochastic independence could also be seen as a fairly accurate description of an ordinary voter's beliefs in most political elections.

\section{A Modest Proposal: Ambiguity Aversion and Ab- stention}

We now show that if we allow the voter to be ambiguity averse, we can obtain abstention under stochastic independence. First, we generalize the voter's decision rule, and then we present an example of a voter who strictly prefers to abstain.

\footnotetext{
${ }^{14}$ It is easy to see that $\mu_{3}=\mu_{4}$ can hold in symmetric equilibria of the voting game of Feddersen \& Pesendorfer (1999) if the set of voter types is discrete. Similarly, when the set of states is infinite (say, because it describes a policy position in a Euclidean space), Feddersen \& Pesendorfer (1999) prove in their Corollary 1 that the expected fraction of voters whose beliefs satisfy conditions (ii) and (iii) (hence are such that $\mu_{3} \neq \mu_{4}$ ) converges to 0 as the expected number of voters increases.
} 


\subsection{Our Model}

Our point of departure is the MEU model of Gilboa \& Schmeidler (1989). This model extends the SEU model by allowing the voter's beliefs to be represented by a set of priors, and assuming that he chooses the action that maximizes the smallest possible expected utility with respect to priors in his set. Formally, there is a cardinal utility $u$ and a nonempty, closed and convex set of probability measures on $T$, labelled $\mathcal{P},{ }^{15}$ such that the voter chooses the act $f \in\{a, b, n\}$ which maximizes

$$
U(f)=\min _{\pi \in \mathcal{P}} \int_{T} u(f(t)) d \pi(t) .
$$

Assumption 3 The voter's preferences satisfy the axioms of Gilboa E Schmeidler (1989) (more precisely, those of Ghirardato et al. (2001)). Equivalently, they are represented by the MEU model, with a utility $u$ and "beliefs" $\mathcal{P}$.

While the aversion to ambiguity of these preferences is a bit extreme, we remark that in this model the voter's perception of the quality of information (ambiguity) is measured by the size of the $\mathcal{P}$ set (Ghirardato, Maccheroni \& Marinacci (2002)). The smaller $\mathcal{P}$ is, the smaller is the bearing of the voter's ambiguity attitude in his choices. For instance, when $\mathcal{P}$ is a singleton, the voter $i s$ SEU.

A key premise of the analysis of Feddersen \& Pesendorfer (1996) is that the voter realizes that his vote will affect the election only in the event $r_{3} \cup r_{4}$ that he is pivotal. He thus conditions his beliefs on that event. In the main body of the paper, we also make the assumption that the voter conditions his beliefs on the event that his vote is pivotal. This is formalized as follows: Every $\pi \in \mathcal{P}$ is such that for every $A \subseteq R$, if we denote by $\lambda$ the marginal of $\pi$ on $R$, we have $\lambda(A)=\lambda\left(A \cap\left(r_{3} \cup r_{4}\right)\right)$.

Assumption 4 The voter's beliefs $\mathcal{P}$ are conditioned on the event $\left(r_{3} \cup r_{4}\right)$, in the sense defined above.

Differently from what happens in the SEU model, such conditionalization is not a harmless assumption, since MEU preferences are in general not dynamically consistent. In Section 6 (and more extensively in Appendix B) we show that this assumption can be substantially weakened, and even removed, at little cost for the substance of our results. We employ the present strong formulation in order to depart as little as possible from the SEU model discussed in the previous section. In fact, the SEU model satisfies conditionalization by necessity.

Finally, we have the announced stochastic independence of election results from policy positions. This is not a limitation of our analysis: as in the SEU case, adding the right type of correlation makes abstention more likely. We make this assumption in order to

\footnotetext{
${ }^{15}$ Closed in the so-called "weak* topology" on $\Delta(T)$. Because in such topology the set $\mathcal{P}$ is compact, the "min" in Eq. (3) is well-defined.
} 
better outline the effects of introducing ambiguity aversion in the voter's choice model. We use the following extension of stochastic independence for MEU preferences (see, e.g., Gilboa \& Schmeidler (1989)). Given two nonempty, closed and convex sets $\mathcal{L}$ and $\mathcal{M}$ of probabilities on $R$ and $S$ respectively, we let

$$
\mathcal{P}=\operatorname{conv}(\{\lambda \times \mu \in \Delta(T): \lambda \in \mathcal{L}, \mu \in \mathcal{M}\}) \equiv \mathcal{L} \otimes \mathcal{M}
$$

That is, the set $\mathcal{P}$ is the closed convex hull generated by all the product measures generated by taking a measure from $\mathcal{L}$ and a measure from $\mathcal{M}$.

Assumption 5 The voter's beliefs $\mathcal{P}$ are an independent "product" $\mathcal{L} \otimes \mathcal{M}$.

When this assumption holds, the voter chooses $f \in\{a, b, n\}$ to maximize the following objective function: ${ }^{16}$

$$
U(f)=\min _{\substack{\lambda \in \mathcal{L} \\ \mu \in \mathcal{M}}} \int_{R} \int_{S} u\left(f\left(r, s_{1}, s_{2}\right)\right) d \mu\left(s_{1}, s_{2}\right) d \lambda(r) .
$$

Hence, the voter evaluates any one of his choices according to a probabilistic belief which reflects stochastic independence of $R$ from $S$, even though these beliefs may change from option to option. Notice that we are not making any assumptions on the form of $\mu$. In particular, we are not assuming that the voter sees the candidates' policy choices as stochastically independent.

\subsection{Abstention Can Be Strictly Optimal: An Example}

Consider a voter who satisfies assumptions 1-3 and 5. As above, given a prior $\mu$ on the candidates' policy positions, we let $\mu^{A}$ (resp. $\mu^{B}$ ) denote the marginal of $\mu$ on its first (resp. second) coordinate. From Table 2 and Eq. (4) we see that our voter's $U(a)$ can be written as follows:

$$
U(a)=\min _{\substack{\lambda \in \mathcal{L} \\ \mu \in \mathcal{M}}}\left[\left(\lambda_{1}+\lambda_{2}+\lambda_{3}+\lambda_{4}\right) v\left(\mu^{A}\right)+\lambda_{5} v\left(\mu^{B}\right)\right]
$$

where for every $j \in\{A, B\}, v\left(\mu^{j}\right)$ is defined by Eq. (2). (That is, $v\left(\mu^{j}\right)$ is the voter's expected utility of having candidate $j$ in office according to the prior $\mu$.) Similarly, we can rewrite $U(n)$ and $U(b)$ as follows:

$$
\begin{aligned}
U(n) & =\min _{\substack{\lambda \in \mathcal{L} \\
\mu \in \mathcal{M}}}\left[\left(\lambda_{1}+\lambda_{2}+\lambda_{3}\right) v\left(\mu^{A}\right)+\left(\lambda_{4}+\lambda_{5}\right) v\left(\mu^{B}\right)\right], \\
U(b) & =\min _{\substack{\lambda \in \mathcal{L} \\
\mu \in \mathcal{M}}}\left[\left(\lambda_{1}+\lambda_{2}\right) v\left(\mu^{A}\right)+\left(\lambda_{3}+\lambda_{4}+\lambda_{5}\right) v\left(\mu^{B}\right)\right] .
\end{aligned}
$$

\footnotetext{
${ }^{16}$ The proof of this claim is available from the authors upon request.
} 
We let $V(\mathcal{M})$ denote the set of the possible pairs $\left(v\left(\mu^{A}\right), v\left(\mu^{B}\right)\right)$ of expected utilities of the candidates as we let $\mu \in \mathcal{M}$ vary. That is,

$$
V(\mathcal{M}) \equiv\left\{\left(v\left(\mu^{A}\right), v\left(\mu^{B}\right)\right) \in[0,1]^{2}: \mu \in \mathcal{M}\right\} .
$$

It follows from assumptions $1-3$ and 5 that for any utility $u, V(\mathcal{M})$ is non-empty, closed, and convex. It is a singleton if $\mathcal{M}=\{\mu\}$; i.e., the voter perceives no ambiguity on the candidates' policy positions. Inspection of (5)-(7) reveals that only a part of $V(\mathcal{M})$ plays a role in the voter's choices: Only those values that correspond to "minima" of linear functions on $V(\mathcal{M})$ are actually used in the voter's decision rule. These belong to the set

$$
E(\mathcal{M}) \equiv\left\{v=\left(v_{1}, v_{2}\right) \in V(\mathcal{M}): v_{1} \geq v_{1}^{\prime} \text { and } v_{2} \geq v_{2}^{\prime}, \text { for no } v^{\prime} \in V(\mathcal{M}), v^{\prime} \neq v\right\} .
$$

$E(\mathcal{M})$ is the locus of the points in $V(\mathcal{M})$ which do not "Pareto dominate" any other point in the set, that we call somewhat imprecisely the "lower envelope" of the set $V(\mathcal{M})$. Figure 1 depicts a typical $V$ and its $E$. (When the set $\mathcal{M}$ is clear, we lighten notation by using $V$ and $E$ instead of $V(\mathcal{M})$ and $E(\mathcal{M})$.)

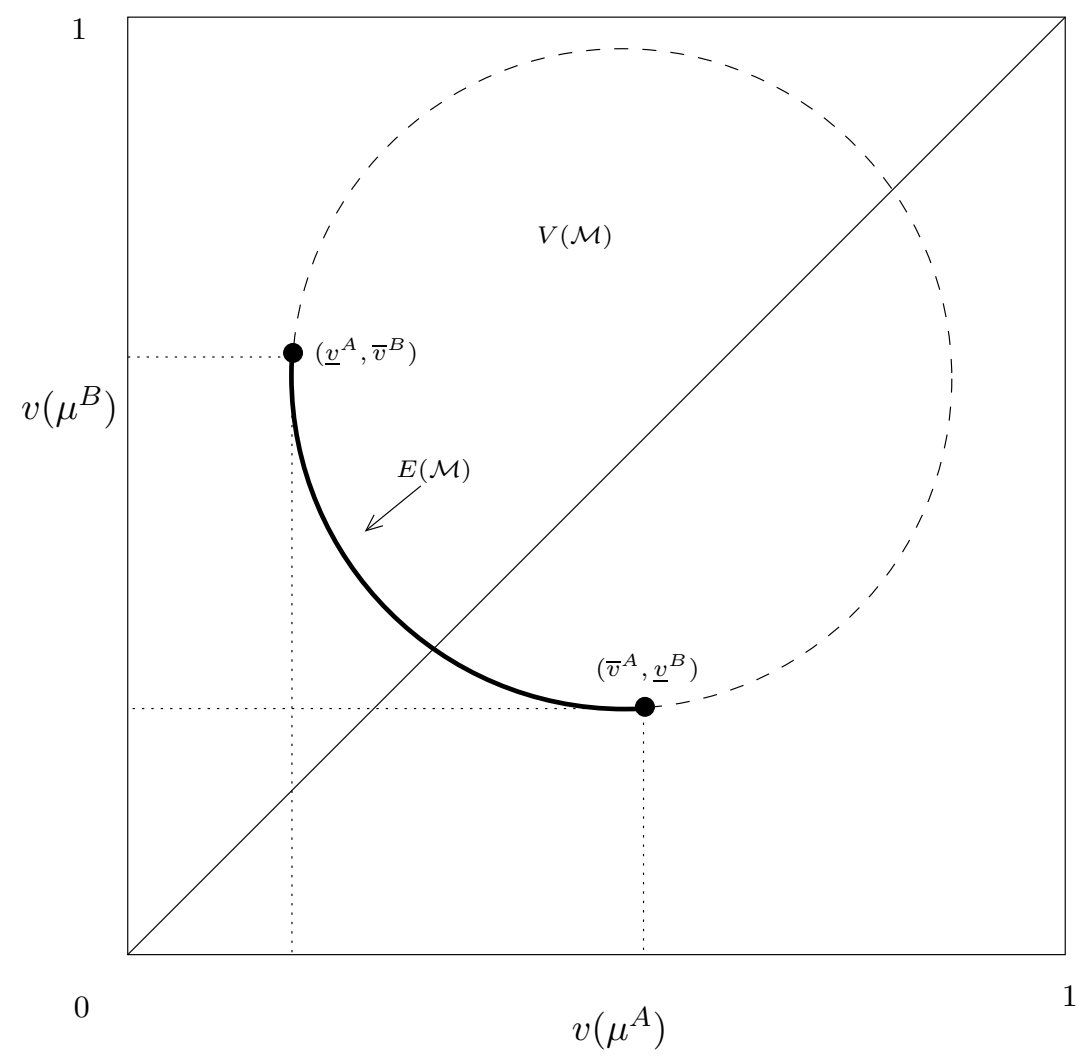

Figure 1: A set $V$ with its "lower envelope" E.

While the voter's beliefs allow for pairs of expected utilities more optimistic than those in the set $E$, the MEU voter behaves as if he only envisions those in $E$ as possible. 
In that sense we refer to the set $E$ as the set of utility pairs that he deems "effective" possibilities. We have drawn $E$ as a decreasing and convex function defined on a closed interval, which is easily shown to be the case. We will henceforth abuse notation, using $E$ to denote both the function and the set it represents (its graph).

When assumption 4 is added, formulas (5)-(7) can be further simplified. As $\lambda_{i}=0$ for all $i \neq 3,4$, every $\lambda \in \mathcal{L}$ can be identified by the single number $\lambda_{3}$, and $\mathcal{L}$ can be reduced to an interval $\mathcal{L}=\left[\ell_{*}, \ell^{*}\right]$. We thus get:

$$
U(a)=\min _{\mu \in \mathcal{M}} v\left(\mu^{A}\right) \equiv \underline{v}^{A}, \quad U(b)=\min _{\mu \in \mathcal{M}} v\left(\mu^{B}\right) \equiv \underline{v}^{B},
$$

and

$$
U(n)=\min _{\mu \in \mathcal{M}}\left\{\begin{array}{lc}
\ell^{*} v\left(\mu^{A}\right)+\left(1-\ell^{*}\right) v\left(\mu^{B}\right) & \text { if } v\left(\mu^{A}\right) \leq v\left(\mu^{B}\right) \\
\ell_{*} v\left(\mu^{A}\right)+\left(1-\ell_{*}\right) v\left(\mu^{B}\right) & \text { otherwise. }
\end{array}\right.
$$

Eq. (8) shows that the two extreme points of $E$ play a special role, as their first and second coordinate $\underline{v}^{A}$ and $\underline{v}^{B}$ are respectively the minimum expected utilities of having $A$ and $B$ in office. Moreover, inspection of Figure 1 reveals that the point minimizing the expected value for $A$ is also the one maximizing the expected value for $B$ over $E$. (This follows from the definition of $E$ and the properties of the set $V$.) Thus the other coordinates of the extremes of $E$, respectively labelled $\bar{v}^{A}$ and $\bar{v}^{B}$, give the "maximum" expected utility that the voter effectively assigns to either candidate. In the figure the minimum and maximum expected utilities for each candidate are different, but this is not universally true: The exceptions are the rectangular $V$, which induce a singleton $E$.

Given $E, \ell_{*}$ and $\ell^{*}$, we can check graphically if abstention is strictly optimal. Consider the voter represented in Figure 2. First, we draw the vertical and horizontal lines dropping to either axis respectively from the points $\left(\underline{v}^{A}, \bar{v}^{B}\right)$ and $\left(\bar{v}^{A}, \underline{v}^{B}\right)$. The intersection of these lines with the diagonal of the unit square tells us which candidate has higher minimum expected utility. For the set $E$ in the figure $\underline{v}^{B}>\underline{v}^{A}$. Thus, this voter would prefer to vote for $B$ than to vote for $A$. As for the minimum expected utility of abstention, Eq. (9) implies that $U(n)$ can be read on the graph as follows: Construct the family of piecewise linear functions with slope $-\ell^{*} /\left(1-\ell^{*}\right)$ above the diagonal and $-\ell_{*} /\left(1-\ell_{*}\right)$ below the diagonal (clearly if $\ell_{*}=\ell^{*}$ these two slopes coincide); find the leftmost of these functions which intersects $E$, and then look at its intersection with the diagonal. If this intersection is strictly to the right of the intersection of the two dashed lines with the diagonal, abstention is strictly optimal. For the voter in the figure abstention is strictly optimal.

In the next section we attempt to provide a clear intuition as to why a voter may end up looking like the one depicted here. Roughly, this happens because the voter perceives the two candidates as ambiguous prospects whose ambiguity is "complementary" in the sense that in those scenarios in which candidate $A$ looks bad, candidate $B$ looks good, and vice versa. The voter is thus afraid that voting for either candidate will turn out to be a mistake (he will find out that the other candidate was better). On the other hand, 


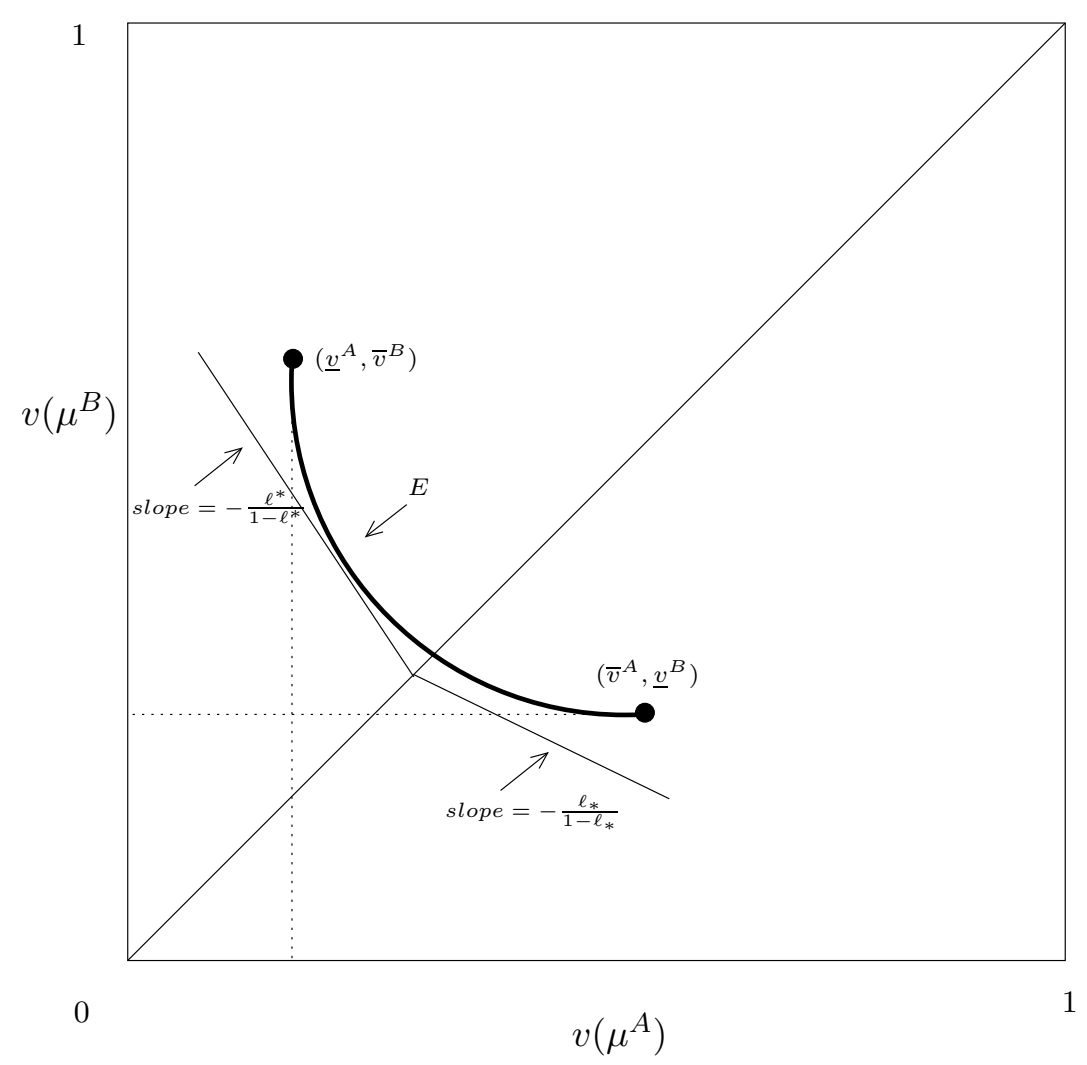

Figure 2: A voter who strictly prefers to abstain.

abstention offers a way of "hedging" the ambiguity of the two candidates by "mixing" between them, thereby smoothing the voter's payoff profile.

It should be remarked from the outset that while ambiguity aversion is a necessary ingredient in our explanation of abstention, it will be found to be not sufficient. In particular, it is not generally true that increasing ambiguity aversion, in the natural sense of enlarging the set of priors, makes abstention more likely. What is needed beyond ambiguity aversion is that the voter perceives the two candidates as sufficiently "complementary", so that he can use abstention to hedge his prospects.

\section{The "Psychology" of Abstention under MEU}

In this section we flesh out more fully our explanation for a strict preference for abstention, both from an intuitive and from a formal perspective.

Let $x^{j}$ be the random variable on $S$ representing candidate $j$ 's policy position. It 
follows from Eq. (8) that for each $f \in\{a, b\}$,

$$
U(f)=\min _{\mu \in \mathcal{M}} v\left(\mu^{j(f)}\right)=\min _{\mu \in \mathcal{M}} \int_{S} u\left(x^{j}(s)\right) d \mu(s),
$$

where $j(f)=A$ if $f=a$, and is $B$ otherwise. That is, we can equivalently represent the voter's evaluation of his options as follows: Voting for candidate $j$ amounts to facing the ambiguous prospect $x^{j}$, which the voter evaluates by taking the "worst" prior in the set $\mathcal{M}$. On the other hand, Eq. (9) implies that abstaining amounts to facing a "mixed" prospect $\gamma x^{A}+(1-\gamma) x^{B}$ for some $\gamma \in(0,1) .{ }^{17}$

The voter's preference over mixed prospects can be represented by the functional $U^{m}$ defined as follows: for any $\gamma \in[0,1]$ and prospects $x$ and $x^{\prime}$, let

$$
U^{m}\left(\gamma x+(1-\gamma) x^{\prime}\right) \equiv \min _{\mu \in \mathcal{M}}\left[\gamma \int_{S} u(x(s)) d \mu(s)+(1-\gamma) \int_{S} u\left(x^{\prime}(s)\right) d \mu(s)\right]
$$

Clearly, such $U^{m}$ is concave. Hence, for the given $x^{A}$ and $x^{B}$,

$$
U^{m}\left(\gamma x^{A}+(1-\gamma) x^{B}\right) \geq \gamma U^{m}\left(x^{A}\right)+(1-\gamma) U^{m}\left(x^{B}\right)=\gamma U(a)+(1-\gamma) U(b) .
$$

In particular, when $U(a)=U(b)$, Eq. (11) implies that

$$
U^{m}\left(\gamma x^{A}+(1-\gamma) x^{B}\right) \geq U(a)=U(b)
$$

This is a property of MEU preferences that goes by the name of ambiguity hedging. ${ }^{18}$ That is, an agent who displays ambiguity hedging when indifferent among two (ambiguous) prospects may prefer a mixture of them, as the latter may provide the possibility of smoothing (i.e., hedging) the utility profile of the outcomes. Notice that, differently from what is commonly believed, this property is unrelated to a preference for objective randomization. The mixture may be the result, as in our case, of external circumstances to which the agent may not be able to attach "objective probabilities", but he would nonetheless display ambiguity hedging (see Ghirardato et al. (2001) for further discussion).

Ambiguity hedging provides an intuitive explanation of why the voter would have a weak preference for abstention. However, that is not enough: We are interested in understanding why the voter strictly prefers to abstain. It is useful to start by looking at some necessary conditions for the optimality of abstention, which only involve the extreme points of $E, \ell_{*}$ and $\ell^{*}$.

\footnotetext{
${ }^{17}$ Mixtures of random prospects are defined state by state so as to satisfy the corresponding utility equality. That is, for every $s \in S,\left(\gamma x+(1-\gamma) x^{\prime}\right)(s)$ is chosen so that

$$
u\left(\left(\gamma x+(1-\gamma) x^{\prime}\right)(s)\right)=\gamma u(x(s))+(1-\gamma) u\left(x^{\prime}(s)\right) .
$$

${ }^{18}$ It is an axiom in Gilboa \& Schmeidler (1989), who call this property "uncertainty aversion". We do not use such terminology, as we think that the term uncertainty should be used less narrowly, and that the alternative "ambiguity aversion" is better used to describe a more general behavioral trait than ambiguity hedging (see Ghirardato \& Marinacci (2002) for further discussion).
} 
Proposition 2 Suppose that assumptions 1-5 hold. Then for abstention to be strictly optimal for a voter, it is necessary that his sets $\mathcal{L}$ and $\mathcal{M}$ are such that $\ell_{*}, \ell^{*}$, and $\underline{v}^{A}, \underline{v}^{B}, \bar{v}^{A}, \bar{v}^{B}$ satisfy:

$$
\left[\ell_{*}, \ell^{*}\right] \subseteq(0,1)
$$

and

$$
\underline{v}^{A}<\bar{v}^{B} \quad \text { and } \quad \underline{v}^{B}<\bar{v}^{A} .
$$

In fact, the following tighter condition must hold:

$$
\max \left\{\underline{v}^{A}, \underline{v}^{B}\right\}<\min \left\{\left(\ell^{*} \underline{v}^{A}+\left(1-\ell^{*}\right) \bar{v}^{B}\right),\left(\ell_{*} \bar{v}^{A}+\left(1-\ell_{*}\right) \underline{v}^{B}\right)\right\} .
$$

The proof of Eq. (14) follows from the observation that its right-hand side is an upper bound to $U(n)$, while its left-hand side is the utility the voter expects from the best candidate. In terms of Figure 2: for abstention to be preferred to voting for $B$, the straight lines passing through $\left(\underline{v}^{A}, \bar{v}^{B}\right)$ and $\left(\bar{v}^{A}, \underline{v}^{B}\right)$ with respective slopes $-\ell^{*} /\left(1-\ell^{*}\right)$ and $-\ell_{*} /\left(1-\ell_{*}\right)$ have to cross the diagonal strictly to the right of $\left(\underline{v}^{B}, \underline{v}^{B}\right)$. The first two equations follow immediately from Eq. (14).

Intuitively, the proposition says that for abstention to be strictly preferred to voting, two pre-conditions must be met: First, we see in Eq. (12) that the voter must not be extremely ambiguity averse about election results and he must not have extreme beliefs about any one of the pivotal events (e.g., think that only one is really possible, cf. condition (i) in Proposition 1). ${ }^{19}$ The comparative ignorance hypothesis assumption discussed in the introduction suggests that this condition is likely to be satisfied. In fact, the differences in the information about the possible turnout and result (remember that we are assuming that the voter conditions on being pivotal) across elections will likely be not as salient as the differences in information about the candidates' policy positions.

Second, Eq. (13) establishes that the voter must see the two candidates as loosely speaking "negatively correlated": when he evaluates a vote for $A$, he implicitly thinks that $B$ is a superior choice, and vice versa when he evaluates a vote for $B$. Using the notation established at the beginning of the section, we formalize this type of "correlation" as follows. We say that two prospects $x$ and $x^{\prime}$ are ambiguity complements if there exists some $\gamma \in(0,1)$ such that the mixture of $x$ and $x^{\prime}$ with probability $\gamma$ is preferred to getting the best of the two. That is, if say $U^{m}(x) \geq U^{m}\left(x^{\prime}\right)$, we have

$$
U^{m}\left(\gamma x+(1-\gamma) x^{\prime}\right)>U^{m}(x) .
$$

Intuitively, when $x$ and $x^{\prime}$ are ambiguity complements, they perform differently enough in the scenarios that the voter contemplates, so that there exists a mixture of $x$ and $x^{\prime}$

${ }^{19}$ Indeed, Eq. (14) imposes tighter bounds on the ambiguity over $R$ than (12). We must have:

$$
\frac{\underline{v}^{A}-\underline{v}^{B}}{\bar{v}^{A}-\underline{v}^{B}}<\ell_{*} \quad \text { if } \quad \underline{v}^{A}>\underline{v}^{B}, \quad \text { and } \quad \ell^{*}<\frac{\bar{v}^{B}-\underline{v}^{B}}{\bar{v}^{B}-\underline{v}^{A}} \quad \text { if } \quad \underline{v}^{A} \leq \underline{v}^{B} .
$$


that provides a sufficiently smoother utility profile. Now, if we identify each candidate $j$ with the ambiguous prospect $x^{j}$ on $S$, Eq. (13) is easily shown to be equivalent to the fact that there is $\gamma \in(0,1)$ such that

$$
U^{m}\left(\gamma x^{A}+(1-\gamma) x^{B}\right)>\max \left\{U^{m}\left(x^{A}\right), U^{m}\left(x^{B}\right)\right\},
$$

which just says that $x^{A}$ and $x^{B}$ are ambiguity complements in the sense just defined. Notice that while ambiguity hedging allows the possibility that two prospects are ambiguity complements, it does not imply that there are two such prospects.

Graphically, Eq. (13) says that the points $\left(\underline{v}^{A}, \bar{v}^{B}\right)$ and $\left(\bar{v}^{A}, \underline{v}^{B}\right)$ are respectively strictly above and below the diagonal. If, in contrast, $E$ is all to one side of the diagonal, voting for one candidate is strictly optimal. For instance, if $E$ is all below the diagonal, voting for $A$ is optimal. Moreover, Eq. (13) implies that $E$ cannot be a singleton, so that $\underline{v}^{A}<\bar{v}^{A}$ and $\underline{v}^{B}<\bar{v}^{B}$; i.e., the voter perceives ambiguity about both candidates. Ambiguity aversion only on the set of election results cannot (under assumption 5) explain a strict preference for abstention.

There is a sense in which the necessary conditions in the proposition are almost sufficient for a voter (satisfying assumptions 1-5) to strictly prefer abstention. Consider a set $\mathcal{L}$ which satisfies (12). Then it is easy to find a set $\mathcal{M}$ such that the voter with beliefs given by $\mathcal{L} \otimes \mathcal{M}$ strictly prefers to abstain. Similarly, given a set $\mathcal{M}$ such that the extreme points of its $E$ satisfy (13), there are $\ell_{*}$ and $\ell^{*}$ such that the voter with beliefs $\mathcal{L} \otimes \mathcal{M}$ strictly prefers to abstain.

\subsection{Symmetric Beliefs}

We now briefly consider a special case of the choice model presented in the previous section, which strikes us as a natural possibility in all those elections in which the voter has little information. The special case is described by the following:

Assumption 6 Given any pair $\alpha, \beta \in \Delta(X)$, if there is $\mu \in \mathcal{M}$ such that $\mu^{A}=\alpha$ and $\mu^{B}=\beta$, there is $\nu \in \mathcal{M}$ such that $\nu^{A}=\beta$ and $\nu^{B}=\alpha$.

That is, the voter's beliefs have symmetric ambiguity about the candidates' policy positions. If he has a prior which assigns a distribution $\alpha$ to $A$ 's policy choice and $\beta$ to $B$ 's policy choice, he has a prior in which the roles of $A$ and $B$ are reversed.

When the voter's beliefs satisfy this symmetry condition the necessary conditions of Proposition 2 are sufficient:

Proposition 3 Suppose that assumptions 1-6 hold. Then abstention is strictly optimal for the voter if and only if his $\mathcal{L}$ and $\mathcal{M}$ induce $\ell_{*}, \ell^{*}$, and $\underline{v}^{A}, \underline{v}^{B}, \bar{v}^{A}, \bar{v}^{B}$ which satisfy Eqs. (12) and (13). 
To see why sufficiency holds, look back at Figure 2 and imagine translating the set $E$ slightly to the right, so that it is symmetric around the diagonal (as it follows from assumption 6) and $\underline{v}^{A}=\underline{v}^{B}$. Because the two extremes must be on either side of the diagonal, and because the function describing $E$ is decreasing and convex, $E$ intersects the diagonal to the right of $\left(\underline{v}^{B}, \underline{v}^{B}\right)$. As before, $U(n)$ can be read by looking at the intersection of the piecewise linear function with the diagonal.

In this case this intersection must fall in the interior of the segment between the intersection of $E$ with the diagonal and the point $\left(\underline{v}^{B}, \underline{v}^{B}\right)$. To see this, we use the $U^{m}$ on mixed prospects defined by Eq. (10) to rewrite $U(n)$ as follows:

$$
U(n)=\min \left\{U^{m}\left(\ell_{*} x^{A}+\left(1-\ell_{*}\right) x^{B}\right), U^{m}\left(\ell^{*} x^{A}+\left(1-\ell^{*}\right) x^{B}\right)\right\} .
$$

Because of ambiguity hedging and of the symmetry of $\mathcal{M}$, for both $\gamma=\ell_{*}$ and $\gamma=\ell^{*}$ we have

$$
U^{m}\left(\gamma x^{A}+(1-\gamma) x^{B}\right) \geq U^{m}\left(x^{A}\right)=U^{m}\left(x^{B}\right)=\underline{v}^{B},
$$

so that $U(n) \geq \underline{v}^{B}$. Moreover, the inequality has to be strict. In fact, if $U(n)=\underline{v}^{B}$, then since $\ell^{*}<1$ and $\ell_{*}>0$ and $E$ is not a singleton, $E$ contains a point $v$ such that either $v_{1}<\underline{v}^{A}$ or $v_{2}<\underline{v}^{B}$. But either of these possibilities contradicts the fact that the function describing $E$ is convex and decreasing (graphically: the set $E$ must lie above and to the right of the dashed lines).

The above argument should clarify that the result of Proposition 3 does not require the full strength of assumption 6 . In fact, the only consequence of symmetry we used for proving the proposition is that $\underline{v}^{A}=\underline{v}^{B}$. Thus, it is immediate to generalize the proposition as follows: if assumptions 1-5 hold and the voter finds voting for $A$ or $B$ equally attractive, then conditions in Eqs. (12) and (13) are necessary and sufficient for abstention to be strictly optimal.

\subsection{The Asymmetric Case}

When beliefs do not treat the two candidates equally, so that $\underline{v}^{A} \neq \underline{v}^{B}$, the necessary conditions of Proposition 2 are in general not sufficient for abstention to be strictly preferred to voting for the favored candidate. Unlike the symmetric case, where any degree of ambiguity complementarity suffices to generate abstention, in this case the strength of complementarity is key. This is illustrated in Figure 3, where we see a profile $E$ and a pair $\ell_{*}, \ell^{*}$ which satisfy the necessary conditions yet are such that voting for $B$ is strictly preferred to abstaining.

Recall that a MEU voter can use different beliefs from $\mathcal{M}$ in evaluating every action. In this case, the beliefs the voter uses in evaluating abstention are as pessimistic as the beliefs he uses in evaluating voting for either candidate. Intuitively, he does not see the candidates as strongly ambiguity complementary. Abstention does not provide an ambiguity hedge, as it entails a probability $\ell^{*}$ of electing $A$ - who in any scenario that the voter contemplates is significantly worse than $B$. 


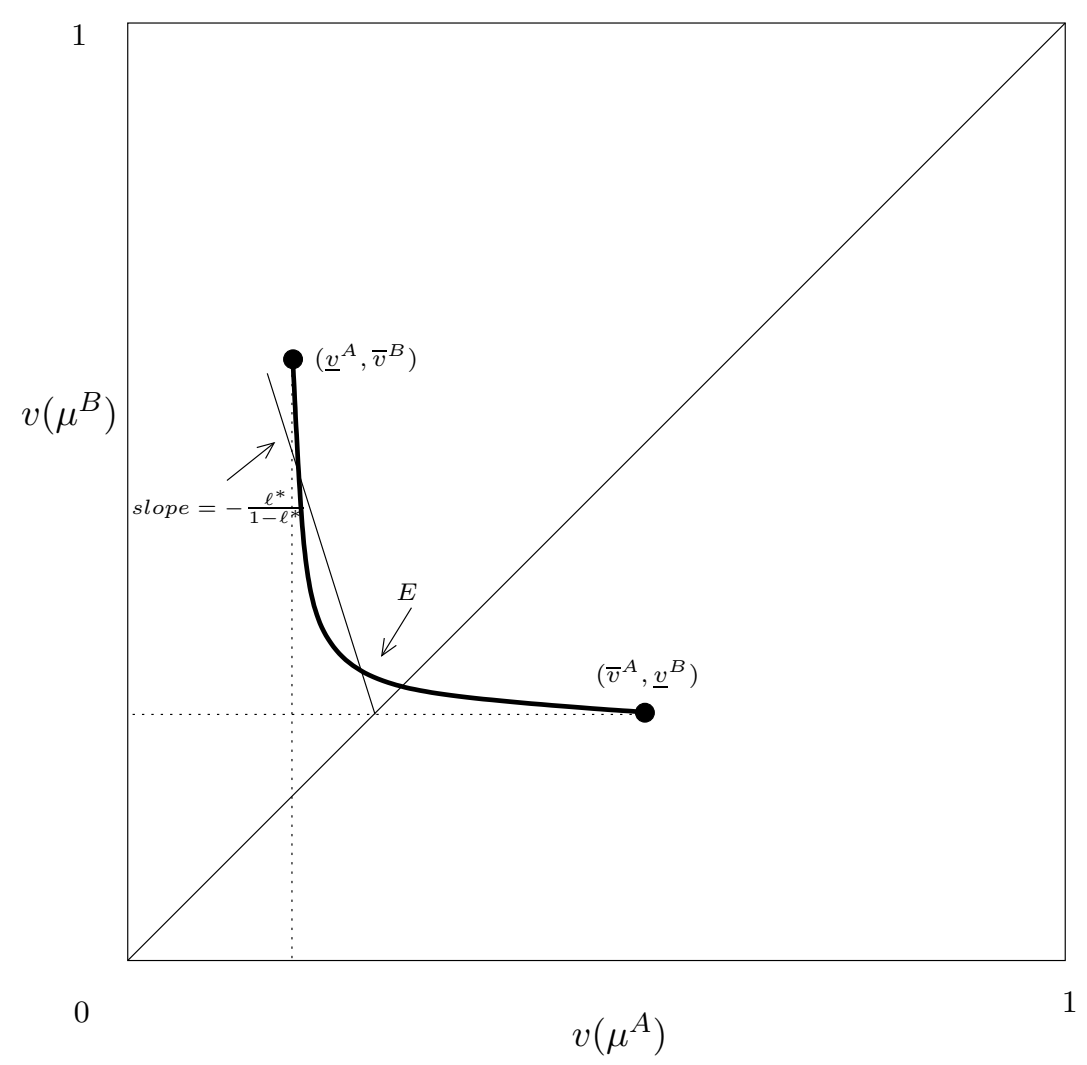

Figure 3: A voter who does not prefer to abstain.

Graphically, this weak complementarity is reflected by the fact that the point of the $E$ curve with slope $-\ell^{*} /\left(1-\ell^{*}\right)$ (not depicted in the figure) is quite "close" to the point $\left(\underline{v}^{A}, \underline{v}^{B}\right)$ at the intersection of the two dashed lines, and it is to the left of the line with the same slope passing through the point $\left(\underline{v}^{B}, \underline{v}^{B}\right)$. Compare this to what happens in Figure 2. There, in evaluating abstention the voter uses beliefs assigning expected utility to candidate $B$ significantly higher than $\underline{v}^{B}$, and expected utility to $A$ a bit higher than $\underline{v}^{A}$. Graphically, the point of $E$ with the required slope (depicted in the figure) is not as "close" to $\left(\underline{v}^{A}, \underline{v}^{B}\right)$ as the one in Figure 3 .

Presenting necessary and sufficient conditions for the optimality of abstention for this case entails the construction of a notion of closeness of the set $E$ to the point $\left(\underline{v}^{A}, \underline{v}^{B}\right)$. The details of this are tedious, and we therefore relegate a result with these conditions to Appendix A (where it appears as Proposition 4).

However, it is easy to show that for any voter who satisfies the necessary condition of Eq. (14), it is always possible to increase the candidates' "degree" of ambiguity complementarity (see the formal definition below) so that the voter will eventually strictly prefer to abstain. ${ }^{20}$

\footnotetext{
${ }^{20}$ The details are available from the authors upon request.
} 


\subsection{Some Comparative Statics}

In closing the analysis of MEU preferences with a strict preference for abstention, we briefly discuss two natural comparative statics exercises. In the first, we fix $\mathcal{L}$ and compare the propensity to abstain of two voters with different sets of priors $\mathcal{M}_{1}$ and $\mathcal{M}_{2}$ on $S$. In the second, we fix $\mathcal{M}$ and compare the propensity to abstain of two voters with different sets of priors $\mathcal{L}_{1}$ and $\mathcal{L}_{2}$ on $R$. In both cases, the voters have a given utility function $u$.

The first exercise requires a formal definition of the relative degree of ambiguity complementarity perceived by two different agents. Given two prospects $x$ and $x^{\prime}$, we say that $\mathcal{M}_{1}$ perceives stronger complementarity between $x$ and $x^{\prime}$ than $\mathcal{M}_{2}$ if for every $\gamma \in(0,1)$,

$$
U_{2}^{m}\left(\gamma x+(1-\gamma) x^{\prime}\right)>U_{2}^{m}(x) \Longrightarrow \gamma U_{1}^{m}\left(\gamma x+(1-\gamma) x^{\prime}\right)>U_{1}^{m}(x)
$$

Said differently: the set of the $\gamma^{\prime}$ s such that $U_{2}^{m}\left(\gamma x+(1-\gamma) x^{\prime}\right)>U_{2}^{m}(x)$ is contained in the set of $\gamma$ 's for which the same strict inequality holds for $U_{1}^{m} ; \mathcal{M}_{1}$ sees more hedging opportunities than $\mathcal{M}_{2}$. Suppose that $\mathcal{M}_{1}$ and $\mathcal{M}_{2}$ are sets of priors such that, say, $U_{i}^{m}\left(x^{A}\right) \geq U_{i}^{m}\left(x^{B}\right)$ for $i=1,2$, and that $\mathcal{M}_{1}$ perceives stronger complementarity between $x^{A}$ and $x^{B}$ than $\mathcal{M}_{2}$. Then, we can see that if the voter with beliefs $\mathcal{M}_{1}$ does not strictly prefer to abstain, the voter with beliefs $\mathcal{M}_{2}$ does not as well. (But the former may strictly prefer to abstain when the second does not.) A simple sufficient condition for the comparative complementarity ranking is that, letting $E_{i}=E\left(\mathcal{M}_{i}\right), E_{1}$ and $E_{2}$ have identical extreme points and $E_{1}$ pointwise dominates $E_{2}$. Such is the case of the two voters depicted in Figure 4 . There for some values of $\ell^{*}$ voter 1 prefers to abstain, while voter 2 prefers to vote for candidate $B$, and if voter 2 prefers to abstains, voter 1 must as well. The figure also illustrates the earlier observation that "more ambiguity aversion" in the sense of a larger set of priors $\mathcal{M}$ does not necessarily imply a greater propension to abstain. In fact, we just need to consider two sets of priors $\mathcal{M}_{1}$ and $\mathcal{M}_{2}$ which respectively induce the sets $V_{1}$ and $V_{2}$ in the figure and are such that $\mathcal{M}_{1} \subset \mathcal{M}_{2}$.

The second comparative statics exercise is more straightforward. Consider $\mathcal{L}_{1}=$ $\left[\left(\ell_{1}\right)_{*},\left(\ell_{1}\right)^{*}\right] \subseteq(0,1)$ and $\mathcal{L}_{2}=\left[\left(\ell_{2}\right)_{*},\left(\ell_{2}\right)^{*}\right] \subseteq(0,1)$, and given $E$. Then, if $\underline{v}^{A} \geq \underline{v}^{B}$ (resp. $\left.\underline{v}^{A} \leq \underline{v}^{B}\right)$, voter 1 prefers to abstain whenever voter 2 does if $\left(\ell_{2}\right)_{*} \leq\left(\ell_{1}\right)_{*}$ (resp. $\left.\left(\ell_{2}\right)^{*} \geq\left(\ell_{1}\right)^{*}\right)$. In particular, the more ambiguity aversion the set $\mathcal{L}$ reveals (again, in the sense of set containment), the less likely is the voter is to abstain, as this tends to decrease the hedging power of abstention by making it less and less likely that the best candidate wins.

\section{$5 \quad$ Multiple Elections}

We can now return to the voter that we left in the polling booth at the beginning of the paper, and predict what he will do in each of the $M$ elections he is asked to vote on. 


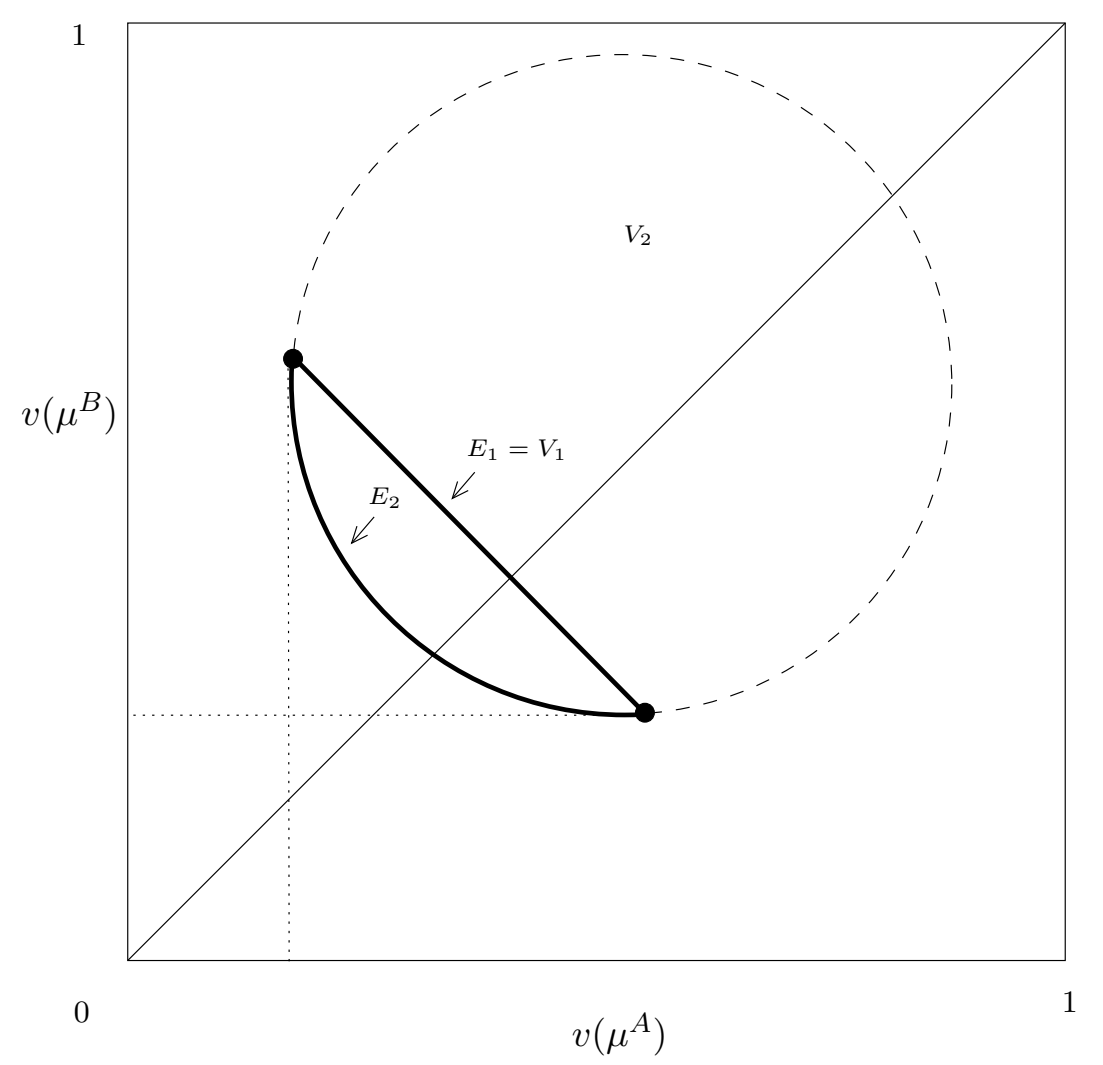

Figure 4: Voter 1 perceives stronger complementarity between $A$ and $B$ than voter 2.

In most circumstances, he will have good information about a subset of elections, little information about another subset, and no information about the remaining ones.

The comparative ignorance hypothesis suggests that the voter displays increasing ambiguity aversion as his information gets poorer. Since abstention can only be optimal if the voter's beliefs induce a $E$ which cuts through the diagonal, it is clear that very little ambiguity aversion (a very small set $V$ ) makes it very unlikely that the voter will abstain. So in general we expect that the voter will not abstain on those elections on which he is well informed. Whether he will abstain on those on which he is poorly informed depends on whether he perceives stronger ambiguity complementarity between the candidates. Is it the case that the lower the relative information the voter has about an election, the stronger the ambiguity complementarity he perceives about the candidates? This is an empirical question that awaits experimental verification, as the experiments of Fox \& Tversky (1995) did not distinguish between ambiguity aversion and ambiguity hedging. Our intuition is that the answer will be positive.

As to those elections on which the voter has no information whatsoever, it seems extremely plausible - again, an assumption that can be experimentally verified - that for those the voter forms symmetric beliefs in the sense of assumption 6 . Thus, he will 
abstain on these elections as long as perceives any complementarity, or equivalently, he is afraid of making a mistake in voting for one of the two candidates.

Summing up, our model predicts that the voter will abstain on those elections on which he sees some ambiguity complementarity. If, as we believe, ambiguity complementarity is inversely related to information, the model explains very well the observed pattern of selective abstention (see also Section 7 below).

\section{Extensions}

In this section we discuss the role that assumptions 1-5 play in the explanation of abstention that we suggest in the foregoing. We show that the model we presented in Section 3 can be extended along most directions, without significantly changing the intuition as to why the voter abstains.

Going in order, assumption 1 is basic to our analysis and it cannot be relaxed. As we observed earlier, it rules out the possibility that the voter might be using his vote as a signal. We have no doubt that signalling could play a role a voter's choice and that it would be useful to extend the analysis of the voter's decision rules in that direction. (It is not obvious to us that such an extension would necessarily provide a rationale for abstaining over voting for a specific candidate.) However, the development of such a model — which would provide a different explanation of abstention - goes well beyond the scope of this paper.

We can "relax" assumption 2 by considering an alternative tie-breaking rule: In case of a tie, a coin is flipped to determine the elected candidate. Because we allow ambiguity averse preferences, some care has to be taken in modeling how the voter treats this coin flip. The most natural assumption (directly implied by the "AnscombeAumann" approach of the seminal paper of Gilboa \& Schmeidler (1989)) is that in any tie-break state the voter uses the "objective" probabilities to calculate the expected utility payoff. For instance, if he votes for $A$ and the election result is $r_{4}$, then his utility is $(1 / 2) v\left(\mu^{A}\right)+(1 / 2) v\left(\mu^{B}\right)$ for some $\mu \in \mathcal{M}$ (that minimizing the overall $U(a)$ ). In that case, we need to modify the conditionalization assumption so that it rules out any $\lambda \in \mathcal{L}$ which assigns positive weight to $r_{1} \cup r_{5}$, since $r_{2}$ is now a pivotal state. It can then be shown (see Appendix $\mathrm{C}$ for details) that, while the conditions for obtaining optimality of abstention may be more demanding than those discussed in Section 4, such behavior may appear in these circumstances for the same reasons discussed in Section 4. If the voter is ambiguity averse about the coin toss, we can reformulate the model by including the result of the coin toss in the description of an election result. The analysis goes through as above. The intuition is identical.

Assumption 3 is clearly crucial for our explanation, so it can be relaxed only partially. A natural weakening of the decision rule we use is that by which the voter evaluates his acts by a convex combination with weight $\alpha \in[0,1]$ of the "min" and the "max" 
(a decision rule inspired by the well-known Hurwicz " $\alpha$-criterion"). It is immediate to see that our results continue to hold for such a model as long as the coefficient $\alpha$ is sufficiently close to 1 . Also, our results can hold verbatim if the voter's preferences satisfy the "Choquet expected utility" model of Schmeidler (1989) — in which the voter's beliefs are represented by a nonadditive probability - if we assume that the voter's non additive belief is "convex", a strong form of superadditivity. While we conjecture that similar results may hold for a more general class of ambiguity averse preferences, here we do not consider further extensions in this direction.

As to assumption 4, it is not necessary for our results. We provide a detailed discussion of this case in Appendix B, where we show that the geometric analysis of the previous sections can be applied after the voter's problem is subjected to a simple transformation. The intuition as to why the voter may abstain is identical to that developed earlier. However, the voter who does not conditionalize may need to perceive stronger complementarity between the candidates in order to prefer abstaining. This is in contrast to what happens in the SEU case. Of course, in the SEU model conditionalization is structural and it cannot really be relaxed, but it plays an important role in explaining why the voter's beliefs satisfy the conditions of Proposition 1. For example, a key conceptual step in the model of Feddersen and Pesendorfer $(1996,1999)$ is that an uninformed voter realizes that his vote matters only when he is pivotal, so that his evaluation of a candidate is made conditionally on his vote being pivotal. A naive voter who does not make this conditioning assumption does not necessarily abstain (of course, he is not playing a symmetric equilibrium of their voting game). For this reason, we think that our explanation of abstention is less driven by conditionalization than those for SEU voters (see Section 7 for additional discussion).

Finally, assumption 5 is not material to our results. As the discussion in Section 2 makes clear, the presence of the right type of correlation in the voter's beliefs can make abstention a strictly optimal choice even without ambiguity aversion. Therefore, when introduced in our model, correlation can intuitively make abstention look even more desirable (it can also make it less desirable, though). Assumption 5 was just made in order to clarify that our explanation of abstention is complementary to those that hinge on correlated beliefs.

\section{Conclusions and Discussion}

In this paper we have taken a decision-theoretic perspective on how information affects a voter's decision to cast a vote on an issue on the election ballot. We showed that if an instrumentally rational voter chooses according to the SEU maximization model, abstention on any of the elections on the ballot is strictly optimal if and only if his beliefs on the candidates' policy positions change significantly across the election results in which his vote is pivotal.

We then introduced considerations of quality of information in the voter's decision 
process, by letting his preferences display ambiguity hedging; i.e., satisfy the MEU model. We showed that in this case abstention is strictly optimal if the voter perceives enough ambiguity complementarity between the candidates, even without any correlation between the voter's beliefs about election results and policy positions. In fact, in that case abstention allows him to hedge the ambiguity in his final utility. We argued that then the voter will abstain on those elections on which he is poorly informed, as long as these "behavioral assumptions" are satisfied: 1) voters perceive higher ambiguity complementarity when more poorly informed, 2) voters form symmetric beliefs when totally uninformed.

It assessing our model, it should be kept in mind that ambiguity hedging and complementarity are properties of preferences that are not related to the existence of external randomizing devices. In particular, we stress that a MEU voter does not necessarily strictly prefer to randomize 1/2:1/2 over two of his actions. As a consequence, flipping a coin to decide which candidate to vote for may not be analogous to abstaining in our model. To most voters, abstention offers a smoother payoff profile than any randomized choice. $^{21}$

The predictions of the model are fully in line with the available evidence. For example, it is well documented that a voter is more likely to vote on those elections for which more money/effort is spent by the candidates, or in general those that are more discussed by the media. Moreover, he is more likely to vote the more educated he is, the longer he has been living in a particular district, and if he is married. ${ }^{22}$ All of these characteristics are indicators for a lower cost of information acquisition. This evidence is only suggestive, as it pertains to the voters' decision of going to the polls, rather than abstaining on selected items once in the voting booth. However, we believe that our model leads to new avenues of empirical research on voting. A natural first step is to find out experimentally if the behavioral assumptions stated above are true. Such experiment will also be helpful for understanding the consequences of ambiguity in a variety of other circumstances. Once the behavioral assumptions are validated, properly designed survey instruments will allow us to compare our model to other explanations of selective abstention.

Having thus summarized the predictions of the voting model that is our main contribution - and indicated a direction in which further work is needed - it is perhaps useful to close the paper with further discussion on the relation between our model and some alternatives, especially in terms of the rationale behind the decision to abstain.

The simplest alternative explanation of selective abstention is that a voter abstains on those elections whose result he does not care about (whether he satisfies SEU or not). The major problem with this explanation is that it is at odds with the observation that voters abstain on elections which they quite obviously care about (e.g., member of the House of Representatives). This is a type of abstention that should be explained by any

\footnotetext{
${ }^{21}$ Indeed, there is abundant experimental evidence (see, e.g., Luce (2000)) that agents "distort" objective probabilities in a fashion that would likely make such course of action less desirable than abstaining.

${ }^{22}$ See, e.g., Wolfinger \& Rosenstone (1980) and Palfrey \& Poole (1987) for US evidence, and Larcinese (2000) for UK evidence.
} 
serious theory of voting behavior. Moreover, we find it hard to name an election that a sophisticated voter would not care about, either directly or vicariously. For instance, a childless voter may still care about the outcome of the school board election — say because he cares about his house's market value, or because he has friends with children.

A second explanation is that proposed by Feddersen and Pesendorfer in $(1996,1999)$. They also study on the role of information in determining the voter's choice, focussing on the role of asymmetries in information across voters. Using a game-theoretic model with SEU voters, they show that some less informed voters will abstain in every (symmetric) equilibrium of the model. Roughly, the reasoning that such voters make in equilibrium is that there are other voters who are more informed, and have similar preferences. Therefore, by abstaining they let the more informed voters make a better decision. While their model undoubtedly captures the reasoning of some very sophisticated voters, we are unsure that it explains the bulk of the empirical evidence. First, because it makes strong requirements on the reasoning ability of every voter (and possibly on the voter's confidence of his understanding of the voting game). Second, because as we mentioned earlier their model assumes a rich set of voter types and a poor set of states, which is not necessarily true in every election. Hence, we think that the model presented here is a useful complement to their model.

A final explanation is the following: Even though the fixed cost of voting is sunk, voting for some candidate entails an additional cost - that of making a reasoned decision - that abstention does not entail. This cost is decreasing in the amount (quality) of information the voter has about the election. Thus, assuming that the voter has a sense of "civic duty" which offsets some small positive cost, the voter will only vote in those elections on which he has enough information. ${ }^{23}$ While there is certainly something to this explanation, it is not fully satisfactory as it begs the main question: Why does abstention carry no decision cost? The explanation of abstention proposed in this paper allows us to rationalize the existence of such a decision cost to voting. A voter who satisfies our assumptions and strictly prefers to abstain behaves as if he perceives an implicit cost to voting, given by the difference between $U(n)$ and the largest of $U(a)$ and $U(b)$. This decision cost is nonnegative because of the ambiguity aversion of the voter, ${ }^{24}$ and it is larger the stronger is the ambiguity complementarity between the candidates. In providing a detailed rationale for the structure of the costs that the decision cost explanation takes as a primitive assumption, our model comes closer to providing a deeper motivation for selective abstention, thus shedding some light on voters' behavior.

\footnotetext{
${ }^{23}$ The model of Matsusaka (1995) offers a dual version of this explanation, where the voter's utility of voting for the correct candidate is increasing in the amount of information the voter has.

${ }^{24}$ It can be seen that it is bounded above by

$$
\min \left\{\ell_{*}\left(\bar{v}^{A}-\underline{v}^{A}\right),\left(1-\ell^{*}\right)\left(\bar{v}^{B}-\underline{v}^{B}\right)\right\},
$$
}

so if the voter is not ambiguity averse about both candidates, the cost cannot be positive. 


\section{Appendix}

\section{Appendix A The Asymmetric Case in Detail}

We begin by introducing a metric in the space of utility pairs. Given $\left(\underline{v}^{A}, \underline{v}^{B}\right)$ and $\left[\ell_{*}, \ell^{*}\right] \subseteq(0,1)$, for any point $v=\left(v_{1}, v_{2}\right)$ in the unit square define

$$
\|v\| \equiv \begin{cases}\left|v_{1}-\underline{v}^{A}\right|+\frac{1-\ell^{*}}{\ell^{*}}\left|v_{2}-\underline{v}^{B}\right| & \text { if } \underline{v}^{A} \leq \underline{v}^{B} \\ \frac{1-\ell_{*}}{\ell_{*}}\left|v_{1}-\underline{v}^{A}\right|+\left|v_{2}-\underline{v}^{B}\right| & \text { otherwise. }\end{cases}
$$

In words, the distance from $v$ to $\left(\underline{v}^{A}, \underline{v}^{B}\right)$ is given by the space that one needs to cover if one is forced only to move along vertical and horizontal lines (for this reason this type of metric is sometimes called the "taxicab" metric). When $\underline{v}^{A} \leq \underline{v}^{B}$ the length of each vertical segment is multiplied by the factor $\left(1-\ell^{*}\right) / \ell^{*}$ (which is equal to 1 when $\ell^{*}=1 / 2$ ). When $\underline{v}^{A}>\underline{v}^{B}$, the length of each horizontal segment is multiplied by $\left(1-\ell_{*}\right) / \ell_{*}$. Notice that $\|v\|=0$ if and only if $v=\left(\underline{v}^{A}, \underline{v}^{B}\right)$.

The following result fully characterizes the class of $\mathcal{L}$ and $\mathcal{M}$ which induce a voter to strictly prefer abstention.

Proposition 4 Suppose that assumptions 1-5 hold. Then abstention is strictly optimal for the voter if and only if his $\mathcal{L}$ satisfies $\left[\ell_{*}, \ell^{*}\right] \subseteq(0,1)$ and for every $v \in E(\mathcal{M})$, with $\|\cdot\|$ defined by Eq. (15), we have:

$$
\|v\|>\max \left\{\left(\underline{v}^{A}-\underline{v}^{B}\right),\left(\underline{v}^{B}-\underline{v}^{A}\right)\right\} .
$$

The proof of this result is easily deduced from Figures 2 and 3. To see why sufficiency holds, assume that $\underline{v}^{A} \leq \underline{v}^{B}$ (the other case is analogous) and notice that if $\left[\ell_{*}, \ell^{*}\right] \subseteq(0,1)$ and Eq. (16) hold, then graphically the curve $E$ is all strictly above the line passing through $\left(\underline{v}^{B}, \underline{v}^{B}\right)$ with slope $-\ell^{*} /\left(1-\ell^{*}\right)$ (which is the locus of the points $v$ such that $\left.\|v\|=\underline{v}^{B}-\underline{v}^{A}\right)$, as depicted in Figure 3. Therefore, the lowest (i.e., leftmost) point of contact of one of the piecewise linear functions with $E$ must be above this line, implying that $U(n)$ is strictly larger than $\underline{v}^{B}$ (see Figure 2). As for necessity, we have already discussed why $\left[\ell_{*}, \ell^{*}\right] \subseteq(0,1)$ is necessary, so suppose that this condition holds and that, contradicting (16), there is a point $v$ on $E$ such that

$$
\|v\| \leq \max \left\{\left(\underline{v}^{A}-\underline{v}^{B}\right),\left(\underline{v}^{B}-\underline{v}^{A}\right)\right\}=\underline{v}^{B}-\underline{v}^{A},
$$

where we assumed as above that $\underline{v}^{B} \geq \underline{v}^{A}$. Now $E$ does intersect the line passing through $\left(\underline{v}^{B}, \underline{v}^{B}\right)$ with slope $-\ell^{*} /\left(1-\ell^{*}\right)$, implying that the minimum expected utility associated with abstention is not larger than $\underline{v}^{B}$ (see Figure 3). This concludes the proof of the proposition. It is also easy to see that both the Propositions 2 and 3 follow from Proposition 4. In fact, condition (14) is clearly implied by the conditions above, whereas when $\underline{v}^{A}=\underline{v}^{B}$ and $\left[\ell_{*}, \ell^{*}\right] \subseteq(0,1)$ hold, Eq. (13) holds iff Eq. (16) holds for every $v \in E$. 


\section{Appendix B The Model without Conditionalization}

Here we show how to extend the model to include voters who do not conditionalize on the event that their vote is pivotal; i.e., they violate assumption 4 .

Given a voter with sets of priors $\mathcal{L}$ and $\mathcal{M}$, define:

$$
\ell a_{*} \equiv \min _{\lambda \in \mathcal{L}}\left[\lambda_{1}+\lambda_{2}+\lambda_{3}+\lambda_{4}\right], \quad \ell n_{*} \equiv \min _{\lambda \in \mathcal{L}}\left[\lambda_{1}+\lambda_{2}+\lambda_{3}\right], \quad \ell b_{*} \equiv \min _{\lambda \in \mathcal{L}}\left[\lambda_{1}+\lambda_{2}\right],
$$

and symmetrically define $\ell a^{*}, \ell n^{*}$ and $\ell b^{*}$ by replacing each "min" with a "max". Using this notation, it follows that Eqs. (5)-(7) are rewritten, for each $f \in\{a, b, n\}$,

$$
U(f)=\min _{\mu \in \mathcal{M}}\left\{\begin{array}{cc}
\ell f^{*} v\left(\mu^{A}\right)+\left(1-\ell f^{*}\right) v\left(\mu^{B}\right) & \text { if } v\left(\mu^{A}\right) \leq v\left(\mu^{B}\right) \\
\ell f_{*} v\left(\mu^{A}\right)+\left(1-\ell f_{*}\right) v\left(\mu^{B}\right) & \text { otherwise. }
\end{array}\right.
$$

One way to characterize the sets of priors which induce abstention in this general case is to construct the set $E$ as before, and study the behavior of the minima of the piecewise linear functions with slope $-\ell f^{*} /\left(1-\ell f^{*}\right)$ above the diagonal, and $-\ell f_{*} /\left(1-\ell f_{*}\right)$ below. But there is a less complex (and less tedious) path. A small modification of the construction in the conditionalization case allows us to directly employ the results obtained for that case. First of all, we modify the set $E$ as follows: For any $v \in E$ let $\varphi(v)$ be the point defined by

$$
\varphi(v) \equiv \begin{cases}{\left[\left(\ell a^{*} v_{1}+\left[1-\ell a^{*}\right] v_{2}\right), v_{2}\right]} & \text { if } v_{1} \leq v_{2} \\ {\left[v_{1}, \ell b_{*}\left(v_{1}+\left[1-\ell b_{*}\right] v_{2}\right)\right]} & \text { otherwise. }\end{cases}
$$

Given the collection of all the points $\varphi(v)$, we remove those points which "Pareto dominate" other points in the collection. ${ }^{25}$ We then call $E^{\prime}$ the resulting set, and let $\left(\left(\underline{v}^{A}\right)^{\prime},\left(\bar{v}^{B}\right)^{\prime}\right)$ and $\left(\left(\bar{v}^{A}\right)^{\prime},\left(\underline{v}^{B}\right)^{\prime}\right)$ denote respectively its left and right endpoints. Like $E, E^{\prime}$ is the graph of a strictly decreasing and convex function on the closed interval $\left[\left(\underline{v}^{A}\right)^{\prime},\left(\bar{v}^{A}\right)^{\prime}\right]$. Next, we transform the probability of election results used to evaluate abstention as follows:

$$
\left(\ell^{*}\right)^{\prime} \equiv \frac{\ell n^{*}}{\ell a^{*}} \quad \text { and } \quad\left(\ell_{*}\right)^{\prime} \equiv 1-\frac{1-\ell n_{*}}{1-\ell b_{*}} .
$$

Figure 5 illustrates a set $E$ with its $E^{\prime}$ and the segment above the diagonal of a level set of $U(n)$ before and after the transformation from $\ell n^{*}$ to $\left(\ell^{*}\right)^{\prime}$.

As a result of this transformation, we face the same geometric problem discussed in Sections 3 and 4 . In particular, the minimum expected utility of candidate $A$ (resp. $B$ ) is found by looking at the ordinate of the left endpoint $\left(\underline{v}^{A}\right)^{\prime}$ (resp. coordinate of the right endpoint $\left.\left(\underline{v}^{B}\right)^{\prime}\right)$ of $E^{\prime}$, and the minimum expected utility of abstention is found by minimizing over $E^{\prime}$ the piecewise linear function with slope $-\left(\ell^{*}\right)^{\prime} /\left[1-\left(\ell^{*}\right)^{\prime}\right]$ above the

\footnotetext{
${ }^{25}$ Such points could exist, as the collection of the $\varphi(v)$ may bend backwards.
} 


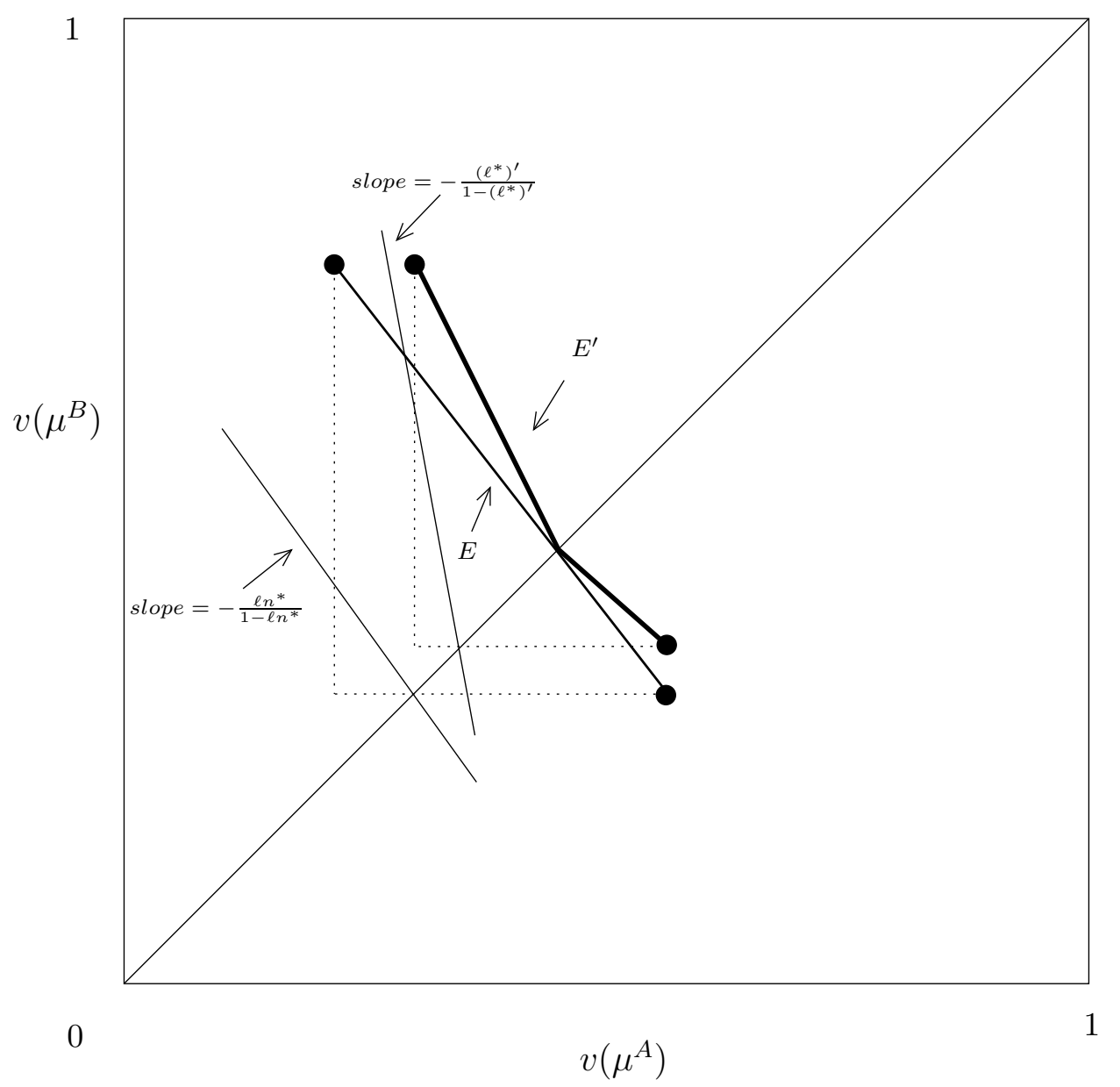

Figure 5: $A$ set $E$ and its modified version $E^{\prime}$.

diagonal, and $-\left(\ell_{*}\right)^{\prime} /\left[1-\left(\ell_{*}\right)^{\prime}\right]$ below. ${ }^{26}$ In light of this observation, we find that all the results and most of the conclusions of Section 4 can be reformulated for this case. ${ }^{27}$

Theorem 1 Suppose that assumptions 1-3 and 5 hold. Propositions 2, 3, and 4 hold once the parameters $\ell^{*}, \ell_{*}, \underline{v}^{A}, \underline{v}^{B}, \bar{v}^{A}, \bar{v}^{B}$ are transformed as explained in the foregoing paragraph (i.e., they are all primed).

(While we have given the rough intuition of the proof of this result, some details have to be taken care of. We thus refer the interested reader to the following subsection for a

\footnotetext{
${ }^{26}$ There is one difference with the analysis in the main text: Here, it may happen that either one of $a$ or $b$ (but not both) is minimized on the "wrong" side of the diagonal. However, we show later that this difference is inconsequential, as it does not affect the necessary and sufficient conditions for abstention.

${ }^{27}$ The exception is the comparative statics of $\mathcal{L}$, as changing the set $\mathcal{L}$ may affect the set $E^{\prime}$, which it also has to be modified. On the other hand, this change reinforces the effects discussed in Subsection 4.3. Thus, it does not alter our conclusion that the more ambiguity averse $\mathcal{L}$ is, the less likely is the voter (with fixed $\mathcal{M}$ ) to abstain.
} 
full proof.)

The first consequence of the theorem is that the mentioned results hold as long as the set $\mathcal{L}$ satisfies

$$
\ell a^{*}=1 \quad \text { and } \quad \ell b_{*}=0 .
$$

In fact, in such a case the transformation is ineffective (primed values are equal to unprimed values). Eq. (18) is equivalent to $\min \lambda_{5}=0$ and $\min \left(\lambda_{1}+\lambda_{2}\right)=0$ : there is some prior in $\mathcal{L}$ which assigns zero probability to $r_{5}$, and some (possibly different) prior which assigns zero probability to $r_{1} \cup r_{2}$. This is significantly weaker than assumption 4 . A stronger version which is still weaker than conditionalization and has an intuitive interpretation is the following: There is a prior $\lambda$ such that $\lambda_{3}+\lambda_{4}=1$. That is, the voter contemplates one scenario in which his vote is certainly pivotal (possibly as well as scenarios in which it is not certainly pivotal). We do not find the latter condition very restrictive. We chose to assume the stronger assumption 4 as a way of better delineating the predictive advantage of the MEU model over the SEU special case.

Recalling the discussion in subsection 4.2, the voter in Figure 5 strictly prefers to abstain, as his transformed effective utility set $E^{\prime}$ does not cross the line with slope $-\left(\ell^{*}\right)^{\prime} /\left(1-\left(\ell^{*}\right)^{\prime}\right)$ passing through the point $\left(\underline{v}^{B}, \underline{v}^{B}\right)$. Thus, the figure shows that there are voters who strictly prefer to abstain even if they do not satisfy assumption 4 or Eq. (18).

However, there is a sense in which the violation of Eq. (18) makes the conditions that guarantee abstention more demanding. To see this more precisely, fix a $\mathcal{M} \subseteq \Delta(S)$ with its effective utilities set $E$ and consider $\mathcal{L}_{1} \subseteq \Delta(R)$ satisfying condition (18). Call voter 1 the voter with these beliefs. Let $\mathcal{L}_{2} \subseteq \Delta(R)$ be a set inducing the same probability weights as $\mathcal{L}_{1}$, except for $\ell a_{2}^{*}$, which satisfies $1=\ell a_{1}^{*}>\ell a_{2}^{*}>\ell n_{1}^{*}=\ell n_{2}^{*}$. Applying the procedure described above to the voter whose beliefs are given by $\mathcal{L}_{2}$ and $\mathcal{M}$, voter 2 , we define $\left(\ell^{*}\right)^{\prime}=\ell n_{1}^{*} / \ell a_{2}^{*}>\ell n_{1}^{*}$, so that above the diagonal the level sets of $U_{2}(n)$ are steeper than those of $U_{1}(n)$. We then transform the section above the diagonal of the set $E$ to obtain the set $E^{\prime}$. Clearly, $E^{\prime}$ is more "bowed in" than $E$, so that voter 2 perceives less complementarity between the candidates than voter 1 . (Again, see Figure 5 for an illustration.) It may thus happen that voter 1 abstains, while voter 2 votes for one candidate ( $A$ in this case).

This difference in behavior is due to the fact that by assigning some positive weight to result $r_{5}$, voter 2 makes voting for $A$ itself a possible ambiguity hedge. Analogously, voting for candidate $B$ may be an ambiguity hedge if $\ell b_{*}>0$. Abstention still provides a more balanced prospect than voting for either candidate, but it yields an ambiguity hedge only if the policy prospects represented by voting for either candidate are sufficiently complementary. Since the latter prospects are themselves mixed, they do not involve the same degree of ambiguity as voting directly for a single candidate's policy position. For this reason, more complementarity between $x^{A}$ and $x^{B}$ is needed for abstention to be preferred in this case. (Roughly, the smaller the difference $\ell a^{*}-\ell b_{*}$, the stronger the complementarity needed.) 
When the voter's beliefs satisfy a stronger version of the symmetry in assumption 6 this difference does not arise. Suppose that the voter's $\mathcal{M}$ set satisfies assumption 6 , and that his $\mathcal{L}$ set is such that $\ell a^{*}=1-\ell b_{*}$. Then it follows from Proposition 5 that he will abstain if and only if $\left[\ell n_{*}, \ell n^{*}\right] \subseteq\left(\ell b_{*}, \ell a^{*}\right)$ and Eq. (13) hold. In this strongly symmetric case the conditions for abstention are essentially independent of whether the voter conditionalizes or not.

Summing up, abstention may be strictly optimal even in the absence of conditionalization or of the weaker condition given in Eq. (18). Moreover, the foregoing discussion has clarified that the intuition we developed as to why a voter strictly prefers to abstain is unaffected by the validity of such assumptions. What may be affected is the strength of the complementarity needed to justify the choice of abstaining.

\section{B.1 Proof of Theorem 1}

To prove this result, we need to show that the following extension of Proposition 4 is true. As both Proposition 2 and 3 are implied by Proposition 4, their "primed" versions then follow analogously.

Proposition 5 Suppose that assumptions 1-3 and 5 hold. Then abstention is strictly optimal for the voter if and only if his $\mathcal{L}$ satisfies $\left[\left(\ell_{*}\right)^{\prime},\left(\ell^{*}\right)^{\prime}\right] \subseteq(0,1)$ and for every $v \in E^{\prime}(\mathcal{M})$, with $\|\cdot\|^{\prime}$ defined by the obvious restatement of Eq. (15), we have:

$$
\|v\|^{\prime}>\max \left\{\left(\underline{v}^{A}\right)^{\prime}-\left(\underline{v}^{B}\right)^{\prime},\left(\underline{v}^{B}\right)^{\prime}-\left(\underline{v}^{A}\right)^{\prime}\right\} .
$$

Proof: Given $\mathcal{L}$ and $\mathcal{M}$ and the probabilities $\ell f^{*}$ and $\ell f_{*}$ defined earlier, we let $E^{+}$and $E^{-}$respectively denote the portions of $E$ weakly above and weakly below the diagonal of the unit square.

We claim that one and only one of the following cases must obtain: ${ }^{28}$

(i) $\{\arg \min U(a)\} \cap E^{+} \neq \emptyset$ and $\{\arg \min U(b)\} \cap E^{-} \neq \emptyset$;

(ii) $\{\arg \min U(b)\} \subseteq E^{+} \backslash E^{-}$and $\{\arg \min U(a)\} \cap E^{+} \neq \emptyset$;

(iii) $\{\arg \min U(a)\} \subseteq E^{-} \backslash E^{+}$and $\{\arg \min U(b)\} \cap E^{-} \neq \emptyset$.

The only part of the claim that needs proof is that cases $(i)-($ iii $)$ are exhaustive. To see this, suppose for example that $\{\arg \min U(b)\} \subseteq E^{+} \backslash E^{-}$. Then it must be the case that

$$
\begin{aligned}
\min _{v \in E^{-}} \ell a_{*} v_{1}+\left(1-\ell a_{*}\right) v_{2} & \geq \min _{v \in E^{-}} \ell b_{*} v_{1}+\left(1-\ell b_{*}\right) v_{2} \\
& \geq \min _{v \in E^{+}} \ell b^{*} v_{1}+\left(1-\ell b^{*}\right) v_{2} \\
& \geq \min _{v \in E^{+}} \ell a^{*} v_{1}+\left(1-\ell a^{*}\right) v_{2}
\end{aligned}
$$

\footnotetext{
${ }^{28}$ In the following we use $\{\arg \min U(f)\}$ to denote the set of the elements of $v \in E$ which attain a minimum of $U(f)$, as defined in Eq. (17).
} 
where we used the fact that $\ell a^{*} \geq \ell b^{*}$ and $\ell a_{*} \geq \ell b_{*}$. Thus, there must be a $v \in$ $\{\arg \min U(a)\} \cap E^{+}$. Similarly, if $\{\arg \min U(a)\} \subseteq E^{-} \backslash E^{+}$, then $\{\arg \min U(b)\} \cap E^{-} \neq$ $\emptyset$.

We now prove sufficiency by looking at each case separately. Consider case $(i)$. Then there are a point $v(a) \in\{\arg \min U(a)\} \cap E^{+}$and a point $v(b) \in\{\arg \min U(b)\} \cap E^{-}$. In this case it follows immediately from the construction of $E^{\prime}$ that

$$
\left(\underline{v}^{A}\right)^{\prime}=\ell a^{*} v(a)_{1}+\left(1-\ell a^{*}\right) v(a)_{2} \quad \text { and } \quad\left(\underline{v}^{B}\right)^{\prime}=\ell b_{*} v(b)_{1}+\left(1-\ell b_{*}\right) v(b)_{2} .
$$

Hence, the extreme points of $E^{\prime}$ assign the correct expected utility to the respective candidate. As to $U(n)$, consider $v \in E^{+}$, and notice that

$$
\begin{aligned}
\left(\ell^{*}\right)^{\prime} \varphi(v)_{1}+\left(1-\left(\ell^{*}\right)^{\prime}\right) \varphi(v)_{2} & =\left(\ell^{*}\right)^{\prime}\left[\ell a^{*} v_{1}+\left(1-\ell a^{*}\right) v_{2}\right]+\left(1-\left(\ell^{*}\right)^{\prime}\right) v_{2} \\
& =\ell n^{*} v_{1}+\left(1-\ell n^{*}\right) v_{2} .
\end{aligned}
$$

Hence

$$
\min _{v \in E^{+}} \ell n^{*} v_{1}+\left(1-\ell n^{*}\right) v_{2}=\min _{v^{\prime} \in\left(E^{\prime}\right)^{+}}\left(\ell^{*}\right)^{\prime} v_{1}^{\prime}+\left(1-\left(\ell^{*}\right)^{\prime}\right) v_{2}^{\prime}
$$

Similarly,

$$
\min _{v \in E^{-}} \ell n_{*} v_{1}+\left(1-\ell n_{*}\right) v_{2}=\min _{v^{\prime} \in\left(E^{\prime}\right)^{-}}\left(\ell_{*}\right)^{\prime} v_{1}^{\prime}+\left(1-\left(\ell_{*}\right)^{\prime}\right) v_{2}^{\prime}
$$

It thus follows that the primed problem and the nonprimed problem assign the same expected utilities $U(f)$ to every $f \in\{a, b, n\}$. Following our argument for Proposition 4, we can now prove that if the conditions stated in the proposition hold, the voter strictly prefers to abstain.

Consider now case $(i i)$. It follows immediately from the fact that $\{\arg \min U(b)\} \subseteq$ $E^{+} \backslash E^{-}$that $\left(\underline{v}^{B}\right)^{\prime}>\left(\underline{v}^{A}\right)^{\prime}$. We need to show that the conditions of the proposition imply the fact that the voter strictly prefers to abstain. In fact this is vacuously true, as in this case Eq. (19) cannot be satisfied. To see this, observe that $\left(\ell^{*}\right)^{\prime} \geq \ell b^{*} / \ell a^{*}$, so that, by the assumption that $\{\arg \min U(b)\} \subseteq E^{+} \backslash E^{-}$, there must be a $v \in\{\arg \min U(b)\}$ such that $\|\varphi(v)\|^{\prime} \leq\left(\underline{v}^{B}\right)^{\prime}-\left(\underline{v}^{A}\right)^{\prime}$. Case (iii) is handled analogously, thus concluding the proof of sufficiency.

Turn to the necessity part. As above, in case $(i)$ we can follow the argument used to prove Proposition 4 to show that a strict preference for abstention implies the conditions in the proposition. What about cases $($ ii $)$ and $($ iii $)$ ? We show that in those cases there is no strict preference for abstention, so that again the implication holds vacuously. To see this in case $(i i)$ (the other case is symmetrical), notice that since $\ell b^{*} \leq \ell n^{*}$, we must have for any $v(b) \in\{\arg \min U(b)\}$,

$$
U(b)=\ell b^{*} v(b)_{1}+\left(1-\ell b^{*}\right) v(b)_{2} \geq \ell n^{*} v(b)_{1}+\left(1-\ell n^{*}\right) v(b)_{2} \geq U(n) .
$$

This concludes the proof of the proposition. 


\section{Appendix C The Coin Flip Tie-Breaking Rule}

As discussed in the text, we first modify the conditionalization assumption so that it rules out any $\lambda$ assigning positive weight to $r_{1} \cup r_{5}$. With this modification, Eq. (17) holds for every $f \in\{a, b, n\}$ with the following coefficients:

$$
\ell a_{*} \equiv \min _{\lambda \in \mathcal{L}}\left[\lambda_{2}+\lambda_{3}+\frac{1}{2} \lambda_{4}\right], \quad \ell n_{*} \equiv \min _{\lambda \in \mathcal{L}}\left[\lambda_{2}+\frac{1}{2} \lambda_{3}\right], \quad \ell b_{*} \equiv \min _{\lambda \in \mathcal{L}}\left[\frac{1}{2} \lambda_{2}\right]
$$

and $\ell a^{*}, \ell n^{*}$, and $\ell b^{*}$ defined as before with a "max" replacing each "min". The analysis is then similar to that in the previous Appendix. Once again, while the conditions for obtaining optimality of abstention might be more stringent than those presented in Section $4,{ }^{29}$ it can appear in these circumstances for the same reasons discussed in Section 4. In the case in which the voter is ambiguity averse about the coin flip, the analysis goes through analogously, with different values for the coefficients $\ell f^{*}$ and $\ell f_{*}$.

\section{References}

Aldrich, John H. 1993. "Rational Choice and Turnout." American Journal of Political Science 37(1):246-278.

Ellsberg, Daniel. 1961. "Risk, Ambiguity, and the Savage Axioms." Quarterly Journal of Economics 75:643-669.

Epstein, Larry G. \& Jianjun Miao. 2000. "A Two-Person Dynamic Equilibrium under Ambiguity." Mimeo, University of Rochester.

Epstein, Larry G. \& Tan Wang. 1994. "Intertemporal Asset Prices under Knightian Uncertainty." Econometrica 62(3):283-322.

Feddersen, Timothy J. \& Wolfgang Pesendorfer. 1996. "The Swing Voter's Curse." American Economic Review 86(3):408-424.

Feddersen, Timothy J. \& Wolfgang Pesendorfer. 1999. "Abstention in Elections with Asymmetric Information and Diverse Preferences." American Political Science Review 93(2):381-398.

Fox, Craig R. \& Amos Tversky. 1995. "Ambiguity Aversion and Comparative Ignorance." Quarterly Journal of Economics 110:585-603.

Ghirardato, Paolo, Fabio Maccheroni \& Massimo Marinacci. 2002. "Ambiguity from the Differential Viewpoint." Mimeo, Caltech, Università Bocconi and Università di Torino.

\footnotetext{
${ }^{29}$ It should be observed that here, in contrast to Appendix B, the differences $\ell a^{*}-\ell n^{*}$ and $\ell n_{*}-\ell b_{*}$ are respectively bounded below by $(1 / 2) \min \left(\lambda_{3}+\lambda_{4}\right)$ and $(1 / 2) \min \left(\lambda_{2}+\lambda_{3}\right)$. Thus, in general we expect the interval $\left[\left(\ell_{*}\right)^{\prime},\left(\ell^{*}\right)^{\prime}\right]$ to be smaller than the one we obtain by relaxing assumption 4 .
} 
Ghirardato, Paolo, Fabio Maccheroni, Massimo Marinacci \& Marciano Siniscalchi. 2001. A Subjective Spin on Roulette Wheels. Social Science Working Paper 1127 Caltech.

Ghirardato, Paolo \& Massimo Marinacci. 2002. "Ambiguity Made Precise: A Comparative Foundation." Journal of Economic Theory 102:251-289.

Gilboa, Itzhak \& David Schmeidler. 1989. "Maxmin Expected Utility with a Non-Unique Prior." Journal of Mathematical Economics 18:141-153.

Hansen, Lars Peter, Thomas J. Sargent \& Thomas D. Jr. Tallarini. 1999. "Robust Permanent Income and Pricing." Review of Economic Studies 66:873-907.

Larcinese, Valentino. 2000. "Information Acquisition and Electoral Turnout: Theory and Evidence from Britain." Mimeo, London School of Economics.

Luce, R. Duncan. 2000. Utility of Gains and Losses: Measurement-Theoretical and Experimental Approaches. London: Lawrence Erlbaum.

Matsusaka, John G. 1995. "Explaining Voter Turnout Patterns: An Information Theory." Public Choice 84:91-117.

Mukerji, Sujoy. 1998. "Ambiguity Aversion and Incompleteness of Contractual Form." American Economic Review 88(5):1207-1231.

Palfrey, Thomas R. \& Keith T. Poole. 1987. "The Relationship Between Information, Ideology, and Voting Behavior." American Journal of Political Science 31:511-530.

Riker, William H. \& Peter C. Ordeshook. 1968. "A Theory of the Calculus of Voting." American Political Science Review 62(1):25-42.

Savage, Leonard J. 1954. The Foundations of Statistics. 1972 revised ed. New York: Dover.

Schmeidler, David. 1989. "Subjective Probability and Expected Utility without Additivity." Econometrica 57:571-587.

Wolfinger, Raymond E. \& Stephen J. Rosenstone. 1980. Who Votes? New Haven, CT: Yale University Press. 